\title{
A dual-targeted molecular therapy of PP242 and cetuximab plays an anti-tumor effect through EGFR downstream signaling pathways in colorectal cancer
}

\author{
Linghui Kong ${ }^{1}$, Qun Zhang ${ }^{1}$, Jialei Mao ${ }^{2}$, Lei Cheng ${ }^{3}$, Xiao Shi ${ }^{1}$, Lixia Yu ${ }^{1}$, Jing $\mathrm{Hu}^{1}$, Mi Yang ${ }^{1}, \mathrm{Li} \mathrm{Li}^{1}$, \\ Baorui Liu ${ }^{1}$, Xiaoping Qian ${ }^{1 \wedge}$ \\ ${ }^{1}$ The Comprehensive Cancer Centre of Nanjing Drum Tower Hospital, The Affiliated Hospital of Nanjing University Medical School, Nanjing, \\ China; ${ }^{2}$ The Comprehensive Cancer Centre of Nanjing Drum Tower Hospital, Clinical College of Nanjing University of Chinese Medicine, \\ Nanjing, China; ${ }^{3}$ Department of Pulmonary Medicine, Shanghai Chest Hospital, Shanghai Jiao Tong University, Shanghai, China \\ Contributions: (I) Conception and design: L Kong, X Qian, B Liu; (II) Administrative support: X Qian, B Liu; (III) Provision of study materials or \\ patients: L Kong, Q Zhang, L Li, L Cheng; (IV) Collection and assembly of data: J Mao, L Yu, J Hu; (V) Data analysis and interpretation: L Cheng, \\ X Shi, M Yang; (VI) Manuscript writing: All authors; (VII) Final approval of manuscript: All authors. \\ Correspondence to: Xiaoping Qian. The Comprehensive Cancer Centre of Nanjing Drum Tower Hospital, The Affiliated Hospital of Nanjing \\ University Medical School, 321 Zhongshan Rd., Nanjing 210008, China. Email: xiaopingqian@nju.edu.cn.
}

Background: Epidermal growth factor receptor (EGFR) and its downstream Ras-mitogen-activated protein kinase kinase (MAPKK, MEK)-extracellular regulated protein kinase (ERK) signaling pathway and phosphatidylinositol 3-kinase (PI3K)-protein kinase B (Akt)-mammalian target of rapamycin (mTOR) signaling pathway play important roles in the pathogenesis of colorectal cancer (CRC). The combination therapy of anti-EGFR and anti-mTOR needs to be explored.

Methods: Here we combined the anti-EGFR monoclonal antibody cetuximab (CTX) with the mTOR inhibitor PP242 in CRC cell lines and mouse xenograft models and discussed the changes of EGFR downstream signaling pathways of CRC cell lines.

Results: In HT-29 cells and Caco-2 cells, combined application of CTX and PP242 significantly inhibited the proliferation of CRC cells in vivo and in vitro. In BRAF wild-type Caco-2 cells, combined application of CTX and PP242 inhibited the activation of the EGFR and its downstream signaling pathways.

Conclusions: Our research further demonstrates the effectiveness of the combined application of CTX and PP242 in inhibiting CRC cell lines from the perspective of cell proliferation, cell cycle, apoptosis, and mouse xenografts. We revealed that the combined application of CTX and PP242 can inhibit tumor growth and proliferation by inhibiting the phosphorylation of key molecules in EGFR downstream MEK-ERK and MEK 4/7 (MKK)-c-Jun N-terminal kinase (JNK) signaling pathways in BRAF wild-type CRC cells. In addition, we found that in BRAF mutant CRC cells, the monotherapy of PP242 resulted in negative feedback increased EGFR phosphorylation rates, accompanied by significant up-regulation of downstream MEK and ERK phosphorylation.

Keywords: Colorectal cancer (CRC); epidermal growth factor receptor (EGFR); mammalian target of rapamycin (mTOR); targeted molecular therapy; signal transduction

Submitted Jun 29, 2021. Accepted for publication Aug 18, 2021.

doi: 10.21037/jgo-21-467

View this article at: https://dx.doi.org/10.21037/jgo-21-467

^ ORCID: 0000-0002-5025-5269.

(c) Journal of Gastrointestinal Oncology. All rights reserved. 


\section{Introduction}

Colorectal cancer (CRC) is a common malignancy in Europe, the Americas, and Oceania. In the United States, the incidence and mortality of CRC ranks third among all malignancies (1), while in China, the disease is common, and its incidence is increasing year by year (2). The treatment of metastatic CRC (mCRC) is comprehensive and includes chemotherapy, radiotherapy, and other modalities. However, the traditional treatment effect of $\mathrm{mCRC}$ is not satisfactory, and its 5 -year survival rate is only $3 \%$ (3). In recent years, the application of molecular targeted agents has greatly improved the survival of mCRC patients (4). Anti-vascular endothelial growth factor (VEGF) monoclonal antibody $(\mathrm{mAb})$ bevacizumab improved survival in mCRC patients by inhibiting tumor angiogenesis (5), and small-molecule inhibitors such as regorafenib and fruquintinib have been shown to be effective in patients with advanced CRC $(3,6)$. Anti-programmed death-1 (PD-1) agents have brought new hope for survival in patients with $\mathrm{mCRC}(7)$, and for $R A S$ and $B R A F$ wild type patients, anti-epidermal growth factor receptor (EGFR) mAb cetuximab (CTX) improved the survival of patients with the disease (8). However, for patients with $B R A F$ mutations, the survival rate is greatly reduced compared with $B R A F$ wild-type patients (9). In CRC, $B R A F$ mutation led to ectopic expression of EGFR (10), reducing the benefit of anti-EGFR therapy (11), and even for $R A S$ wild-type patients, anti-EGFR therapy may lead to the emergence of drug-resistance and clinical relapse (12). As the efficacy of targeted molecular monotherapies is limited by the molecular mechanism of the drugs themselves, new strategies for $\mathrm{mCRC}$ are required.

In recent years, dual-targeted molecular therapy of malignant tumors has been widely applied, providing new hope for prolonging the survival of tumor patients. In CRC, there are also several combined targeted therapies undergoing clinical and preclinical research. The BEACON clinical trials have shown that the combined use of binimetinib, encorafenib, and CTX in patients with mCRC who have failed first-line treatment achieved good results and has been written into the National Comprehensive Cancer Network (NCCN) guidelines (13). In a study of $B R A F$ V600E mutate CRC, the combined application of the BRAF V600E inhibitor vemurafenib, ErbB2 inhibitor afatinib, and anti-EGFR panitumumab showed a good response on CRC cell lines that overexpressed ErbB2 (14). Dual-targeted molecular therapy targeting novel signaling pathways have also received widespread attention in the study of CRC cell lines. A study using a combination of the sterol regulatory element-binding transcription factor 2 (SREBF2) inhibitor dipyridamole and mitogenactivated protein kinase kinase (MAPKK, MEK) inhibitor trametinib (15), and a study using bromodomain-containing protein 4 (BRD4) inhibitor AZD5153 and poly-(ADPribose) polymerase (PARP) inhibitor BMN673 (16) have both reported good results in vivo and in vitro. This research demonstrated the promise of combined targeted therapy in extending the survival of patients with mCRC.

The EGFR signaling pathway has been shown to play an important role in the pathogenesis of CRC (17). EGFR activation affected cell proliferation and differentiation through the Ras-MEK-extracellular regulated protein kinase (ERK) signaling pathway and the phosphatidylinositol 3-kinase (PI3K)-protein kinase B (Akt)-mammalian target of rapamycin (mTOR) signaling pathway (18). In addition, MEK 4/7 (MKK) and its downstream c-Jun N-terminal kinase (JNK), usually considered one of the downstream signaling pathways of EGFR (19), were also closely related to cell proliferation, differentiation, and apoptosis (20). Anti-EGFR therapy is widely used in the treatment of various malignant tumors. In a study of non-small cell lung cancer, the combination of the EGFR inhibitor erlotinib and anti-PD-1 mAb showed good antitumor effects on tumor cells (21), and patients with cutaneous squamous cell carcinoma that was resistant to platinum were effectively treated by anti-EGFR treatment (22). Currently, anti-EGFR CTX is widely used in CRC patients, but the effect is not ideal for some, including those with BRAF mutation (11). There are many reasons for $\mathrm{mCRC}$ resistance to antiEGFR therapies. Mutations in EGFR downstream genes (KRAS, NRAS, BRAF, PIK3CA) can lead to resistance to anti-EGFR therapy in patients with mCRC, which has become a consensus (8). In addition, long-term use of antiEGFR therapy in mCRC patients may develop acquired KRAS mutations and copy number changes such as genes encoding receptor protein tyrosine kinases (RTKs), which are all related to the acquired resistance of antiEGFR therapy (17). Therefore, the application of antiEGFR therapy in patients with CRC still has limitations. Recently, scientists have provided many ideas for reversing the resistance of anti-EGFR therapies, including targeting downstream pathways of EGFR, enhancing the use of cytotoxic drugs, combining anti-angiogenic drugs, inducing oxidative stress, and combining checkpoint inhibitors (23). As an important signaling pathway downstream of EGFR, mTOR-targeted therapy may play an important role in 
reversal of anti-EGFR therapy resistance.

mTOR is an important signaling molecule downstream of the PI3K-Akt signaling pathway, which plays an important role in carcinogenesis. EGFR overexpression in tumor cells led to the activation of the downstream PI3KAkt-mTOR signaling pathway, thereby promoting cell growth, differentiation, and inhibiting apoptosis $(18,24)$. In mammals, activated mTOR forms two multiprotein complexes with other molecules; mTOR complex 1 (mTORC1) and mTOR complex 2 (mTORC2) (25). Both MTORC1 and MTORC2 have different sensitivities to rapamycin, with the former being sensitive and the latter not so due to the presence of the scaffold protein rapamycin insensitive companion of mTOR (26). PP242 is a novel mTOR inhibitor that inhibits both mTORC1 and mTORC2 (27). The application of mTOR inhibitors has been applied in many malignant tumors. In adult T-cell leukemia, dual mTOR inhibitors such as PP242 and AZD8055 inhibited the phosphorylation of Akt and caused tumor cell apoptosis (28), and in platinum-resistant ovarian cancer, the application of PP242 reversed resistance, reduced cell proliferation, and promoted tumor cell killing (29). However, the existence of a negative feedback mechanism reduced the therapeutic efficiency of mTOR inhibitors (30), which may be the reason why PP242 monotherapy cannot be widely promoted. Therefore, we suspect the application of PP242 in CRC cells may lead to negative feedback activation of EGFR, and the combined application of CTX and PP242 may have a better effect than monotherapy.

In our previous work, we found that the combined application of CTX and PP242 inhibited the proliferation and tumor behavior of CRC cell lines and inhibited the tumor growth of tumor xenografts (31). In the present study, we further demonstrated the inhibitory effects of the combined application of CTX and PP242 on the growth of CRC cell lines and mouse xenografts. For the first time, we tested the changes in the signal pathway of CRC cells after the combination of anti-EGFR therapy and mTOR inhibitor PP242, tried to clarify the novel molecular mechanism of the combined targeted therapy, and conducted a preliminary discussion on the changes in these signaling pathways. We present the following article in accordance with the ARRIVE reporting checklist (available at https://dx.doi.org/10.21037/jgo-21-467).

\section{Methods}

\section{Cell lines and cell culture}

EGFR wild-type BRAF mutant HT-29 cells, and BRAF wild-type Caco-2 cells were purchased from the Cell Bank of the Chinese Academy of Sciences (Shanghai, China). HT-29 cells were cultured in Roswell Park Memorial Institute-1640 (RPMI-1640) (Corning, New York, USA) with $10 \%$ fetal bovine serum (FBS) (ExCell Bio, Jiangsu, China), $100 \mathrm{U} / \mathrm{mL}$ penicillin, and $100 \mu \mathrm{g} / \mathrm{mL}$ streptomycin (Pen-Strep) (Beyotime Biotechnology, Shanghai, China). Caco-2 cells were cultured in Dulbecco's modified Eagle's medium (DMEM) (Corning) with 20\% FBS and Pen-Strep. Cells were placed in an incubator at $37^{\circ} \mathrm{C}$, and $5 \%$ carbon dioxide. Culture media was changed every $36 \mathrm{~h}$, and subcultured after reaching about $80 \%$ confluence. For subculturing, cells were washed twice with $2 \mathrm{~mL}$ normal saline (NS) (Baxter, Shanghai, China) and detached with $1 \mathrm{~mL}$ $0.25 \%$ trypsin-ethylene diamine tetraacetic acid (EDTA) (Beyotime Biotechnology) for $3 \mathrm{~min}$ at $37^{\circ} \mathrm{C}$. Cells were then suspended in $1 \mathrm{~mL} \mathrm{FBS}$ and centrifuge at 1,000 rpm for $5 \mathrm{~min}$. The supernatant was discarded, and cells were resuspended with culture media then transferred to another cell culture flask.

\section{Cell counting kit-8 (CCK-8) assay}

HT-29 cells and Caco-2 cells were seeded in a 96-well plate at a density of 1,500 cells per well. Cells were untreated or treated with $20 \mu \mathrm{g} / \mathrm{mL}$ CTX (Merck Serono, Darmstadt, Germany) and $1 \mu \mathrm{mol} / \mathrm{L}$ PP242 (Selleck, Houston, USA) alone or in combination for $96 \mathrm{~h}$ before detection (31). CCK-8 (Vazyme, Jiangsu, China) was used to detect cell proliferation activity, with $10 \mu \mathrm{L}$ CCK- 8 solution added per well. The 96 -well plate was incubated at $37^{\circ} \mathrm{C}$ for $2 \mathrm{~h}$, and the optical density at $450 \mathrm{~nm}(\mathrm{OD} 450)$ was measured to calculate cell proliferation activity.

\section{Cell cycle and cell apoptosis analysis}

Cells were untreated or treated with $20 \mu \mathrm{g} / \mathrm{mL}$ CTX and $1 \mu \mathrm{mol} / \mathrm{L}$ PP242 alone or in combination for $96 \mathrm{~h}$ before detection. A cell cycle staining kit (Multi Sciences, Zhejiang, China) was used to detect the cell cycle and an Annexin V-fluorescein isothiocyanate isomer (FITC)/propidine 
iodide (PI) apoptosis kit (Multi Sciences) was used to detect the cell apoptotic status. Cells were processed according to the instructions and flow cytometry was used to detect their cell cycle and apoptotic status.

\section{Extraction of total cellular proteins and western blot analysis}

Cells were incubated with $100 \mu \mathrm{L}$ radio-immunoprecipitation assay (RIPA) lysate (Beyotime Biotechnology) for $30 \mathrm{~min}$ on ice for complete lysis. For each $10 \mathrm{~mL}$ RIPA lysate, one protease inhibitor cocktail tablet (Roche, Basel, Switzerland) and $100 \mu \mathrm{L}$ phosphatase inhibitor (Solarbio, Beijing, China) was added to prevent degradation of total cellular proteins and phosphorylated proteins. The lysate was centrifuged for $15 \mathrm{~min}$ at $4{ }^{\circ} \mathrm{C}$ at $14,000 \mathrm{rpm}$, then $5 \times$ loading buffer (Beyotime Biotechnology) was added to the supernatant at a volume ratio of $1: 4$. Protein samples were incubated at $95{ }^{\circ} \mathrm{C}$ for $5 \mathrm{~min}$ and stored at $-80^{\circ} \mathrm{C}$. Protein concentration was measured by Quick Start Bradford Protein Assay kit (Bio-Rad, Hercules, USA), and for western blot analysis, equal amounts of cell protein were separated by $15 \%$ sodium dodecyl sulfate polyacrylamide gel electrophoresis (SDS-PAGE). Cell protein was then transferred to a polyvinylidenediflouride (PVDF) membrane (Merck Millipore, Steinheim, Germany) and then blocked with $5 \%$ albumin bovine $\mathrm{V}$ (Aladdin, Shanghai, China). The PVDF membrane was incubated with certain primary antibodies at $4{ }^{\circ} \mathrm{C}$ overnight, then with horse radish peroxidase (HRP)-linked goat anti rabbit immunoglobulin G (IgG) $(1: 50,000)$ (Bioworld Technology, St. Louis, USA) at room temperature for $2 \mathrm{~h}$. The primary antibody and its dilution ratio were as follows: anti- $\beta$-actin rabbit mAb $(1: 5,000)$ (Bioworld Technology); anti-Ki-67 rabbit mAb (1:1,000); anti-platelet endothelial cell adhesion molecule-1 (CD31) rabbit polyclonal antibody (pAb) (1:1,000); anti-caspase-3 rabbit pAb (1:500); anti-cleaved caspase-3 rabbit mAb (1:1,000) (Beyotime Biotechnology); anti-m-TOR rabbit mAb (1:1,000); anti-phospho m-TOR rabbit $\mathrm{mAb}(1: 1,000)$; anti-EGFR rabbit $\mathrm{mAb}(1: 1,000)$; antiphospho EGFR rabbit mAb (1:1,000); anti-MEK 1/2 rabbit mAb (1:1,000); anti-phospho MEK rabbit mAb $(1: 1,000)$, anti-p44/42 ERK rabbit mAb (1:1,000); anti-phospho-p44/42 ERK rabbit mAb (1:2,000); anti phospho-MKK rabbit mAb (1:1,000); anti-JNK rabbit mAb (1:1,000); anti-phospho JNK rabbit mAb $(1: 1,000)$ (Cell Signal Technology, Denver, USA); and anti-MKK rabbit mAb (1:1,000) (Abcam, Cambridge,
UK). $\beta$-actin was used as the endogenous control.

\section{Extraction of total cellular RNA and quantitative real time polymerase chain reaction ( $\mathrm{P} C \mathrm{R}$ )}

Cells were incubated with $1 \mathrm{ml}$ Trizol lysate (Thermo Fisher Scientific, Waltham, USA) for $5 \mathrm{~min}$ on ice for complete lysis. Chloroform (200 $\mu \mathrm{L})$ (Nanjing Reagent, Jiangsu, China) was added to the lysate to dissolve the RNA into the organic phase, followed by centrifuging at $4{ }^{\circ} \mathrm{C}, 14,000 \mathrm{rpm}$ for $15 \mathrm{~min}$. A pipette was used to gently aspirate the upper layer solution and transfer it to another Eppendorf tube, and an equal volume of isopropanol to precipitate the RNA was added before being centrifuged at $4{ }^{\circ} \mathrm{C}, 14,000 \mathrm{rpm}$ for $15 \mathrm{~min}$. The supernatant was discarded, and the sediment washed with $1 \mathrm{~mL} \mathrm{75 \%}$ ethanol [prepared with anhydrous ethanol (Nanjing Reagent) and diethyl pyrocarbonate (DEPC) water (Beyotime Biotechnology) at a ratio of 3:1], and centrifuged at $4^{\circ} \mathrm{C}, 14,000 \mathrm{rpm}$ for $5 \mathrm{~min}$. RNA was dissolved with DEPC water and reverse transcribed into cDNA with a transcriptor first strand cDNA synthesis kit (Roche) according to the instructions. For qPCR reactions, the following reaction system was prepared in a 384-well plate: $5 \mu \mathrm{L}$ SYBR Green PCR Master Mix (Thermo Fisher Scientific), $0.2 \mu \mathrm{L}$ upstream primer, $0.2 \mu \mathrm{L}$ downstream primer, and $1 \mu \mathrm{L} \mathrm{cDNA}$, supplemented with DEPC water to $10 \mu \mathrm{L}$. Each reaction was replicated three times to minimize errors. qPCR reactions were performed under an ABI 7900 System (Thermo Fisher Scientific). Primers were synthesized by Tsingke Biological Technology (Beijing, China) and included the following: forward glyceraldehyde-3-phosphate dehydrogenase (GAPDH): 5'-GGAGCGAGATCCCTCCAAAAT-3', reverse GAPDH: 5'-GGCTGTTGTCATACTTCTCATGG-3', forward EGFR: 5'-TTGCCGCAAAGTGTGTAAC G-3', reverse EGFR: 5'-GTCACCCCTAAATGCCACC G-3', forward MEK: 5'-CAATGGCGGTGTGGTGTT C-3', reverse MEK: 5'-GATTGCGGGTTTGATCTCCA G-3', forward ERK: 5'-TCACACAGGGTTCCTGACAG A-3', reverse ERK: 5'-ATGCAGCCTACAGACCAAATAT C-3', forward MKK: 5'-TGCAGGGTAAACGCAAAGC A-3', reverse MKK: 5'-CTCCTGTAGGATTGGGATTCA GA-3', forward fNK: 5'-TCTGGTATGATCCTTC TGAAGCA-3', and reverse $7 N K$ : 5'-TCCTCCAAGTCC ATAACTTCCTT-3'. GAPDH was used as the endogenous control. 


\section{Small interfering RNA (siRNA), plasmids and cell transfection}

siRNA and plasmids were transfected into cells by Lipofectamine 3000 (Thermo Fisher Scientific), and cells were seeded into 6-well plates at a density of $1.2 \times 10^{5}$ cells per well. Lipofectamine 3000, siRNA, and plasmids were mixed with Opti-MEM (Thermo Fisher Scientific) in a specific ratio according to the manufacturer's instruction. The cell culture was discarded, and Opti-MEM then added to continue culturing the cells. The mixture was slowly added to the supernatant, and the cells further cultured in the incubator for $6 \mathrm{~h}$. The supernatant was then discarded, and the cells continued to be cultured with a serum-containing culture medium, before being collected for further analysis after $36 \mathrm{~h}$. siRNA and EGFR-pcDNA3.1(+) plasmids were purchased from GenePharma (Shanghai, China). Sequences of siRNA were as follows: forward si-negative control (NC): 5 '-UUCUCCGAACGUGUCACGUTT-3', reverse siNC: 5'-ACGUGACACGUUCGGAGAATT-3', forward siEGFR1: 5' - CCGAAAGCCAACAAGGAAATT-3', reverse siEGFR1: 5' - UUUCCUUGUUGGCUUUCGGT T-3', forward siEGFR2: 5'- GCAACAUGUCGAUGGACU UTT-3', and reverse siEGFR2: 5'- AAGUCCAUCGAC AUGUUGCTT-3'.

\section{Tumor xenografts}

PP242 was dissolved with a solvent mixed with 2\% dimethyl sulfoxide (DMSO) (Biofroxx, Einhausen, Germany), 30\% polyethylene glycol (PEG) 400 (Nanjing Reagent), 5\% Tween 80 (Ketai, Jiangsu, China), and 63\% water, and was configured as a solution with a final concentration of $5 \mathrm{mg} / \mathrm{mL}$. HT29 cells were injected subcutaneously into 6-week-old male $\mathrm{BALB} / \mathrm{c}$ nude mice with $5 \times 10^{6}$ cells per mouse, and when the subcutaneous mass of the mice reached about $100 \mathrm{~mm}^{3}$, they were randomly divided into four groups. The control group was treated with NS $(4 \mathrm{~mL} / \mathrm{kg})$ and the solvent $(6 \mathrm{~mL} / \mathrm{kg})$; the CTX group was treated with CTX $(20 \mathrm{mg} / \mathrm{kg})$ and the solvent $(6 \mathrm{~mL} / \mathrm{kg})$; the PP242 group was treated with NS $(4 \mathrm{~mL} / \mathrm{kg})$ and PP242 $(30 \mathrm{mg} / \mathrm{kg})$; and the CTX + PP242 group was simultaneously treated with CTX $(20 \mathrm{mg} / \mathrm{kg})$ and PP242 (30 mg/kg). The body weight and tumor volume of the mice were measured and treatments were given intraperitoneally (32) twice per week. Tumor volume was calculated as length $\times$ width $^{2} / 2$. After 2 weeks of treatment, mice were sacrificed and heart, liver, kidney, and tumor tissues were harvested for further analysis. Experiments were performed under a project license (No.: 2019AE01054) granted by the Nanjing Drum Tower Hospital ethics committee, in compliance with China national or institutional guidelines for the care and use of animals.

\section{Hematoxylin-eosin (HE) staining and immunohistochemistry assay}

Heart, liver, spleen, lung, kidney, and tumor tissues of mice were fixed with $4 \%$ paraformaldehyde solution for at least 24 hours. After dehydration and paraffin embedding, tissues were cut into $5 \mathrm{~mm}$ serial sections and then used for HE staining and immunohistochemical analysis. The section was dewaxed and washed with distilled water. For HE staining, sections were counterstained with HE (Servicebio, Wuhan, China) according to the manufacturer's instructions. For immunohistochemistry assay, the section was blocked with QuickBlock immunostaining bloc (Beyotime Biotechnology) after antigen retrieval. The sections were incubated with the primary antibody and the HRP-labeled secondary antibody successively. A 3,3 N-diaminobenzidine tertrahydrochloride (DAB) Horseradish Peroxidase Color Development Kit (Beyotime Biotechnology) was used to develop color, and after dehydration and sealing with neutral gum, the sections were observed and photographed under a microscope. The primary antibody and its dilution ratio were as follows: antiKi-67 rabbit mAb (1:200); anti-CD31 rabbit pAb (1:200); and anti-cleaved caspase-3 rabbit mAb (1:200).

\section{Statistical analysis}

Data were analyzed with Graphpad prism 8.0.2 (Graphpad Inc.; La Jolla, USA) and are shown as the mean \pm standard error of mean (SEM). Student's $t$-test and one-way analysis of variance (ANOVA) was used for statistical significance among groups. A P value of 0.05 or less was considered statistically significant.

\section{Results}

\section{Combined application of CTX and PP242 inbibited the proliferation of CRC cell lines}

To investigate the effect of the combined application of CTX and PP242 on the proliferation of CRC cell lines, we performed CCK-8 cell proliferation assay on HT-29 and Caco-2 cells. In Caco-2 cells, the combined application 
resulted in a significantly higher cell proliferation inhibition ratio than that in the control group and each monotherapy group $(\mathrm{P}<0.001)$ (Figure 1A). In the HT-29 cells, the cell inhibition rate of the combined treatment group was significantly higher than that of the control group and the CTX monotherapy group $(\mathrm{P}<0.001)$ (Figure $1 B)$. In addition, we detected cell cycle and apoptosis of CRC cell lines by flow cytometry. When compared with the control group, the combined application of CTX and PP242 significantly reduced the proportion of $\mathrm{S}$-phase cells in either HT-29 cells or Caco- 2 cells $(\mathrm{P}=0.042$ and $\mathrm{P}=0.024$ respectively) (Figure $1 C-1 F$ ). Conversely, after the combined therapy, the apoptosis ratio of tumor cells increased significantly compared with the control group $(\mathrm{P}=0.002$ and $\mathrm{P}=0.006$ respectively) (Figure 1G-17). In HT-29 cells, the proportion of S-phase cells decreased significantly, and the proportion of apoptotic cells increased significantly when compared with the CTX monotherapy group $(\mathrm{P}=0.015$ and $\mathrm{P}=0.003)$. Our data showed that the combined application of CTX and PP242 could inhibit the proliferation of CRC cells by altering the cell cycle and promoting apoptosis.

The expression of certain proteins in cells reflects their biological state to a certain extent. Ki-67 is one of the common markers related to cell proliferation and can be used to judge cell proliferation activity (33). CD31 is an endothelial cell-specific protein (34), and the angiogenic tendency of tumors can be determined by detecting its content in cells. The caspase family plays an important role in the initiation and execution of apoptosis. Among the caspases, caspase- 3 can cause apoptosis after being cleaved (35), and the apoptotic status of cells can be reflected by detecting its activation. Therefore, we measured $\mathrm{Ki}-67, \mathrm{CD} 31$, and cleaved caspase-3 in CRC cell lines as biological markers. When compared with the control group, the combined application of CTX and PP242 significantly reduced the expression of $\mathrm{Ki}-67(\mathrm{P}=0.002$ and $\mathrm{P}=0.008$ respectively) and $\mathrm{CD} 31$ ( $\mathrm{P}=0.046$ and $\mathrm{P}=0.030$ respectively), while significantly increasing caspase- 3 activation $(\mathrm{P}=0.023$ and $\mathrm{P}=0.014$ respectively) in both HT-29 cells (Figure S1A,S1B) and Caco-2 cells (Figure S1C,S1D). Our data further illustrated that combination therapy could affect CRC cell proliferation, angiogenesis, and apoptosis.

\section{The combined application of CTX and PP242 has an anti- tumor effect in mouse xenograft models}

To evaluate the effects of the combined application of CTX and PP242 on xenograft tumors in vivo and its toxic and side effects, we constructed subcutaneous xenograft models of HT-29 cells in nude mice and applied CTX and PP242 alone or in combination to them. The combined application of CTX and PP242 significantly reduced the size of subcutaneous xenografts $(\mathrm{P}=0.002)$ without significant changes on the body weight of mice $(\mathrm{P}=0.769)$ (Figure $2 A-2 C$ ). In addition, we harvested the heart, liver, spleen, lung, and kidney tissues of mice for HE staining analysis and found there was no significant difference in HE staining morphology in tissues of the different treated groups, and no lesion morphology was observed in the vital organs of mice (Figure 2D). The above data showed that the combination of CTX and PP242 effectively inhibited the growth of subcutaneous transplanted tumors in mice without significant toxic and side effects.

To further prove that the combined application of CTX and PP242 had an inhibitory effect on mouse subcutaneous xenografts, we sliced and immunostained the mouse subcutaneous xenografts. Consistent with the results of cell experiments, the combined application of CTX and PP242 in xenografts significantly down-regulated the expression of Ki-67 and CD31 ( $\mathrm{P}=0.001$ and $\mathrm{P}=0.002$ respectively) in tumor tissues and up-regulated the activation of caspase- 3 $(\mathrm{P}<0.001)$ (Figure $\mathrm{S} 2)$. The data further illustrated that the combination therapy could affect the growth, angiogenesis, and apoptosis in CRC xenografts.

\section{Application of PP242 inhibited the phosphorylation of mTOR in CRC cells}

To verify the inhibitory effect of PP242 on mTOR, we investigated the phosphorylation of mTOR in CRC cell lines. In HT-29 cells and Caco-2 cells, the application of PP242 significantly reduced the phosphorylation of cellular mTOR ( $\mathrm{P}=0.007$ and $\mathrm{P}=0.011$ respectively) but had no significant effect on the total mTOR content $(\mathrm{P}=0.654$ and $\mathrm{P}=0.338$ respectively) (Figure $\mathrm{S} 3$ ). Our results proved that the mTOR inhibitor PP242 could effectively inhibit the phosphorylation of mTOR.

\section{mTOR inbibitor PP242 up-regulated EGFR gene expression and phosphorylation in BRAF mutant HT-29 cells}

To investigate the molecular changes caused by CTX and PP242 treated alone or in combination to CRC cell lines, we measured the gene expression rates, protein content, and activation of EGFR and key molecules in EGFR 

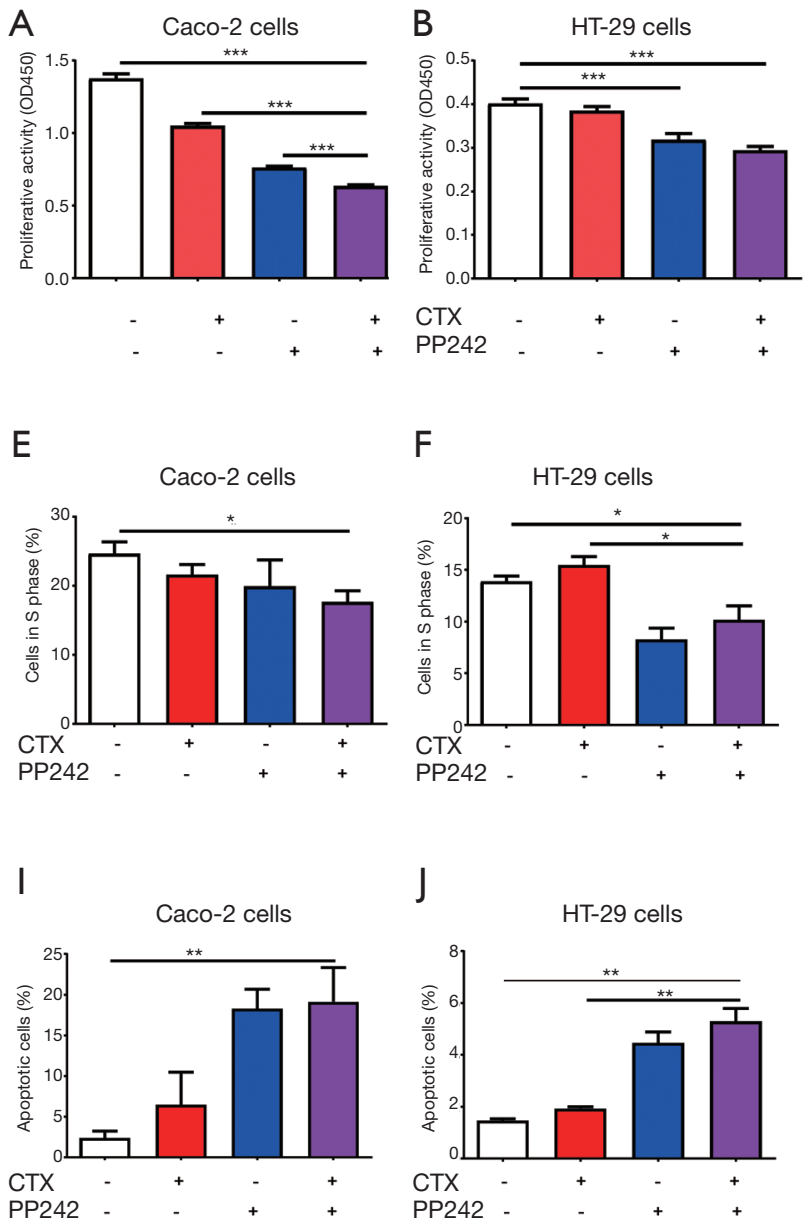

$\mathrm{F}$

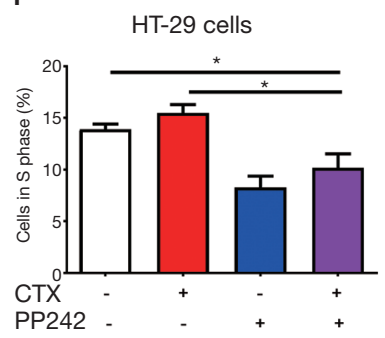

J

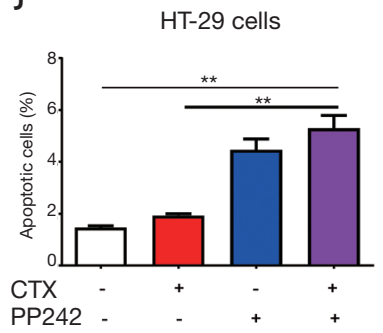

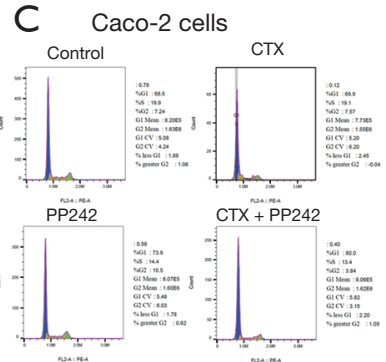

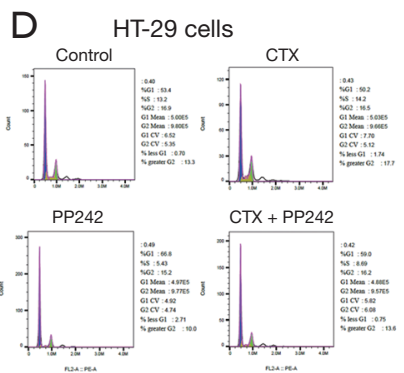

G
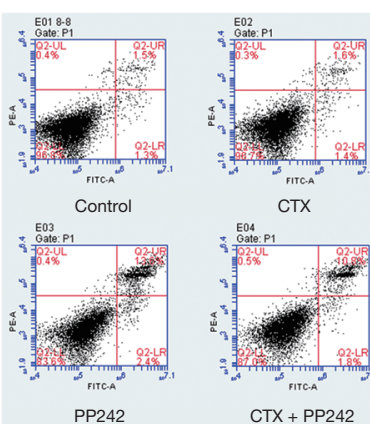

$\mathrm{H}$
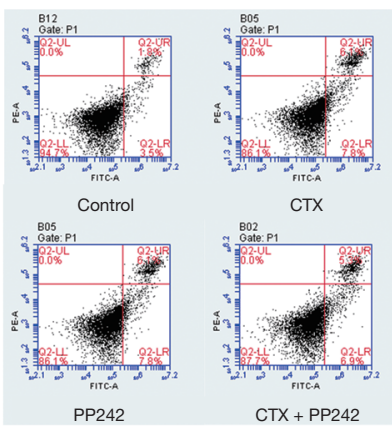

Caco-2 cells

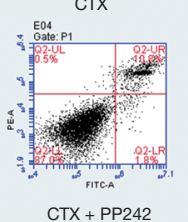

PP242
HT-29 cells

Figure 1 Combined application of CTX and PP242 inhibits the proliferation of CRC cells. (A,B) EGFR wild-type Caco-2 cells (A) and HT-29 cells (B) were untreated or treated with $20 \mu \mathrm{g} / \mathrm{mL}$ CTX and $1 \mu \mathrm{mol} / \mathrm{L}$ PP2 42 alone or in combination for 96 h. Cell viability was determined by the CCK-8 assay and indicated by OD450. (C-F) Flow cytometry was used to detect the cell cycle of CRC cell lines. Caco2 cells (C,E) and HT-29 cells (D,F) were untreated or treated with $20 \mu \mathrm{g} / \mathrm{mL}$ CTX and $1 \mu \mathrm{mol} / \mathrm{L}$ PP242 alone or in combination for 96 h. The relative numbers and proportions of cells in each group at G1, S, and G2 phases are shown in (C) and (D), respectively. The proportion of cells in $\mathrm{S}$ phase was used to assess cell division activity and displayed in (E) and (F). (G-J) Flow cytometry was used to detect the cell apotosis of CRC cell lines. Caco-2 cells (G,I) and HT-29 cells (H,J) were untreated or treated with $20 \mu \mathrm{g} / \mathrm{mL}$ CTX and $1 \mu \mathrm{mol} / \mathrm{L}$ PP242 alone or in combination for $96 \mathrm{~h}$. Different apoptotic states of cells are shown in $(\mathrm{G})$ and $(\mathrm{H})$ and divided into four quadrants. The lower left quadrant, upper left quadrant, lower right quadrant, and upper right quadrant represent normal cells, necrotic cells, early apoptotic cells, and late apoptotic cells, respectively. The proportion of late apoptotic cells was used to assess the status of apoptotic cells and is displayed in (I) and (J). Each bar represents the mean \pm SEM. All data were derived from at least three separate experiments. ${ }^{*}, \mathrm{P}<0.05 ;{ }^{* *}, \mathrm{P}<0.01 ;{ }^{* * *}$, $\mathrm{P}<0.001$. CTX, cetuximab; CRC, colorectal cancer; EGFR, epidermal growth factor receptor; CCK-8, cell counting kit-8; OD450, optical density at $450 \mathrm{~nm}$; SEM, standard error of mean.

downstream signaling pathways. In $B R A F$ mutant HT-29 cells, the application of PP242 significantly increased the gene expression $(\mathrm{P}=0.017)$ and protein content $(\mathrm{P}=0.008)$ of EGFR, which led to a significant increase in phosphorylation of EGFR, MEK, and ERK $(\mathrm{P}<0.001, \mathrm{P}=0.011$ and $\mathrm{P}=0.008$ respectively). When compared with PP242 monotherapy, the phosphorylation of EGFR decreased significantly after the combined application of CTX and PP242 ( $\mathrm{P}=0.031)$ (Figure 3). However, in the detection of the MKK-JNK signaling pathway downstream of EGFR, the combined 
A

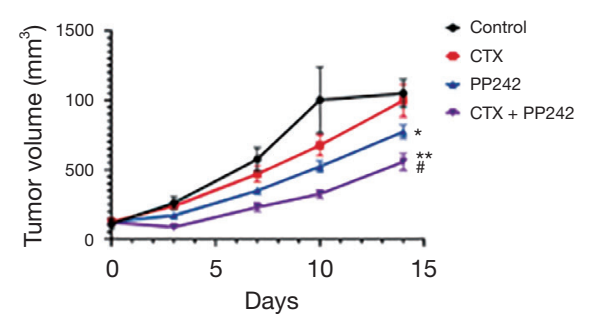

B

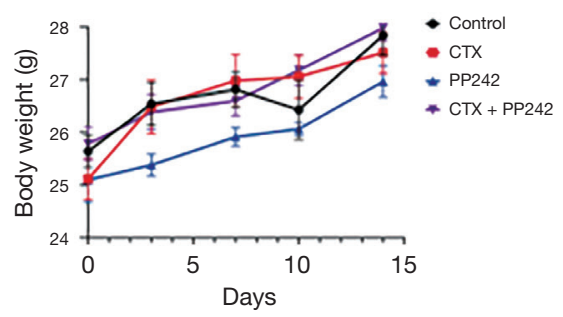

C

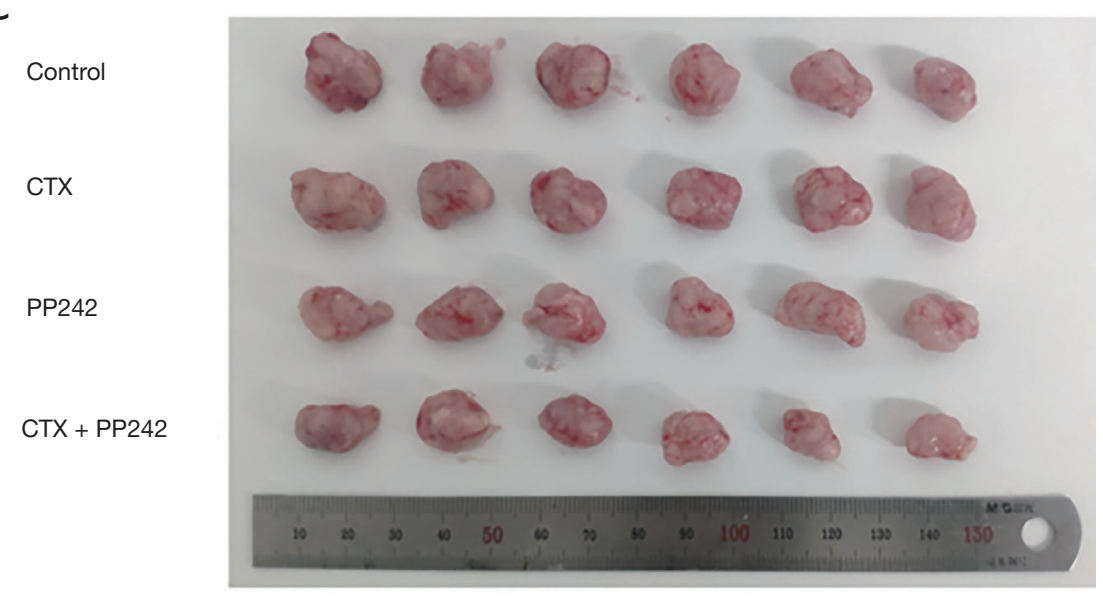

D

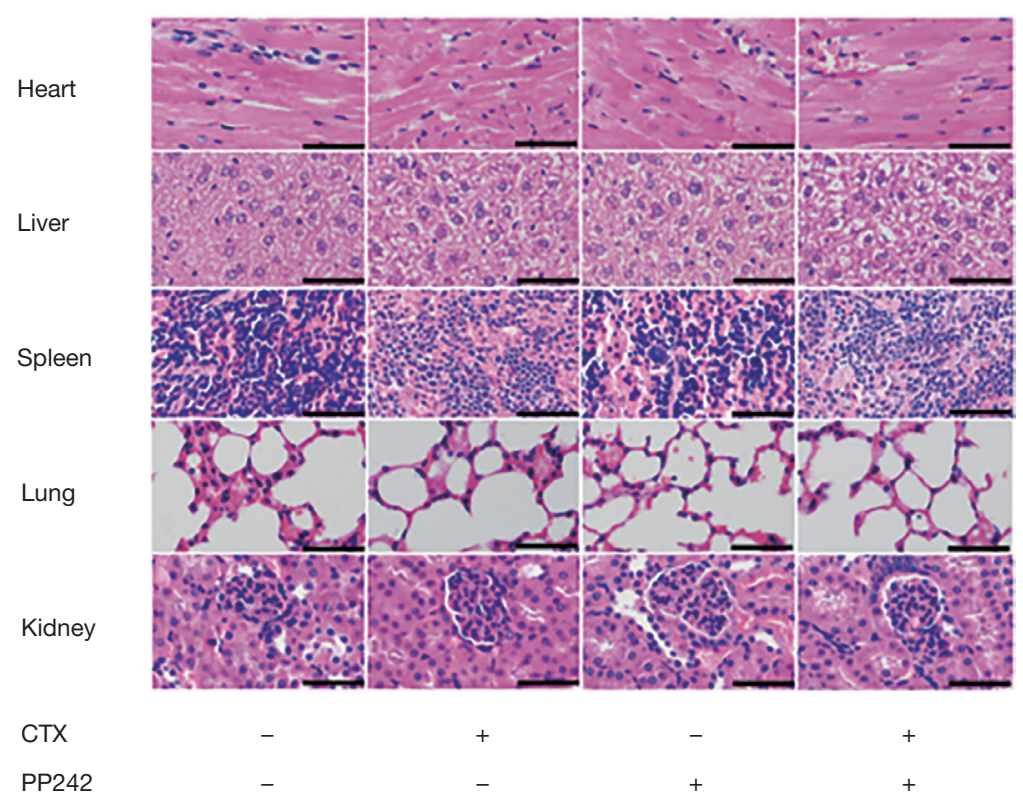

Figure 2 Combined application of CTX and PP242 inhibits the growth of xenograft tumors in mice without significant toxicity. Six-week-old male BALB/c nude mice bearing HT-29 cell xenograft tumors were untreated or treated with $20 \mathrm{mg} / \mathrm{kg}$ CTX and $30 \mathrm{mg} / \mathrm{kg}$ PP242 alone or in combination twice a week. (A) Mouse xenograft tumor volumes during 2 weeks of treatment. (B) Mouse body weights during 2 weeks of treatment. (C) Mouse xenograft tumors from all groups. (D) Microscopic view of HE staining of mouse heart, liver, spleen, lung, and kidney sections. Data are shown as the mean \pm SEM. Scale bar: $50 \mu \mathrm{m} .{ }^{*}, \mathrm{P}<0.05$ and ${ }^{* *}, \mathrm{P}<0.01$ when compared with the control group; ", P<0.05 when compared with the CTX group. CTX, cetuximab; HE, hematoxylin-eosin; SEM, standard error of mean. 


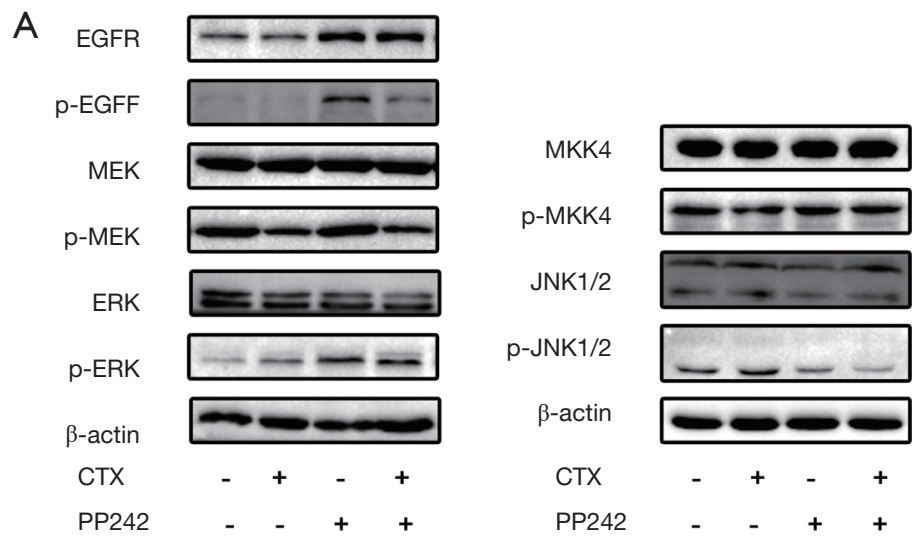

B
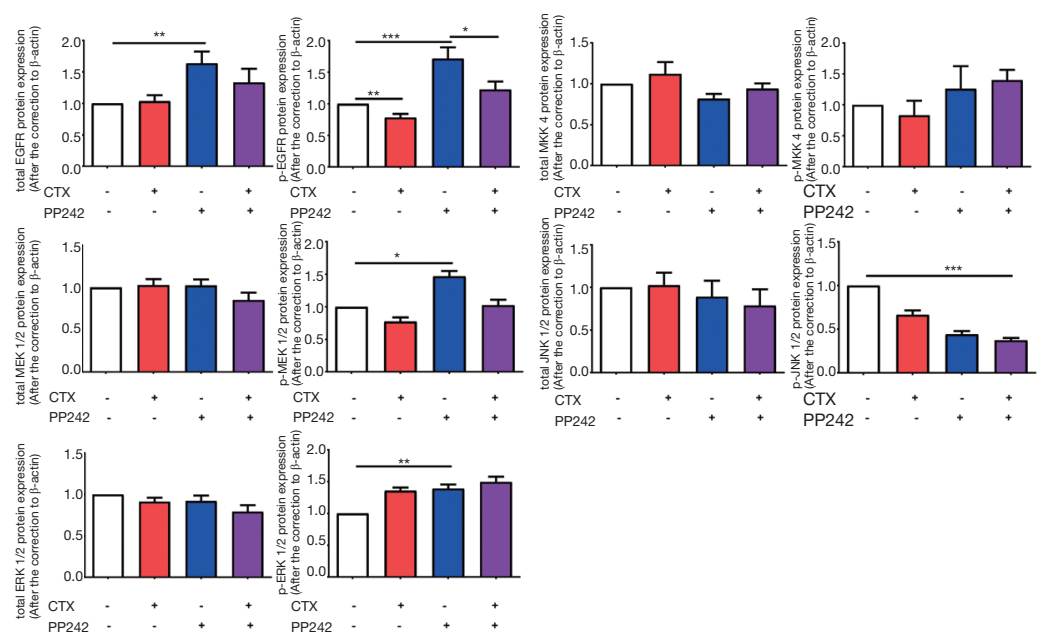

C
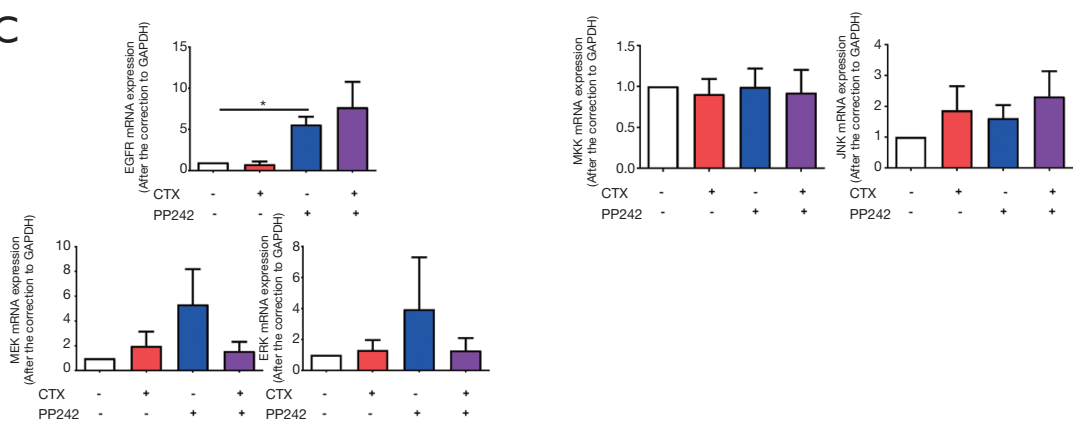

Figure 3 mTOR inhibitor PP242 up-regulated EGFR expression and phosphorylation but had no significant effect on the downstream signaling pathways of EGFR in HT-29 cells. HT-29 cells were untreated or treated with $20 \mu \mathrm{g} / \mathrm{mL} \mathrm{CTX} \mathrm{and} 1 \mu \mathrm{mol} / \mathrm{L}$ PP242 alone or in combination for $96 \mathrm{~h}$. (A) The protein content of cellular total and phosphorylated EGFR, MEK, ERK, MKK and JNK contents were determined by western blotting. (B) The semi-quantitative analysis of cellular total and phosphorylated EGFR, MEK, ERK, MKK and JNK contents was performed based on the relative density values of the bands of western blotting. The relative density value of the control group was set to one. (C) qPCR was used to measure the mRNA expression of EGFR, MEK, ERK, MKK and JNK in different treatment groups relative to the control group. The relative mRNA expression of the control group was set to one. Each bar represents the mean \pm SEM. All data were derived from at least three separate experiments. *, $\mathrm{P}<0.05$; ${ }^{* *}, \mathrm{P}<0.01$; ${ }^{* * *}, \mathrm{P}<0.001$. mTOR, mammalian target of the rapamycin; EGFR, epidermal growth factor receptor; CTX, cetuximab; MEK, mitogen-activated protein kinase kinase; ERK, extracellular regulated protein kinase; MKK, MEK 4/7; JNK, c-Jun N-terminal kinase; qPCR, quantitative real time polymerase chain reaction; SEM, standard error of mean. 
application of HT-29 and PP242 only significantly reduced the phosphorylation of JNK $(\mathrm{P}<0.001)$ and no significant differences were found in the gene expression, protein content of MKK, JNK, and phosphorylation of MKK (Figure 3). Therefore, we speculate that in HT-29 cells, the application of PP242 will negatively up-regulate EGFR expression, which will cause different activation states of key molecules downstream of EGFR.

\section{Combined application of CTX and PP242 down-regulates the EGFR downstream signaling patbways in BRAF wild- type Caco-2 cells}

We also measured the activation of EGFR downstream signaling pathways after combined therapy in $B R A F$ wildtype Caco-2 cells. The results showed that, contrary to the experimental results in HT-29 cells, the combined application of CTX and PP242 significantly reduced the phosphorylation rates of EGFR and downstream MEK and ERK $(\mathrm{P}=0.024, \mathrm{P}=0.018$ and $\mathrm{P}=0.020$ respectively), but had no effect on the gene expression rates and total protein content (Figure 4). In addition, the experimental results of the MKK-JNK signaling pathway downstream of EGFR were similar. The combined application of CTX and PP242 reduced the phosphorylation of $\mathrm{MKK}$ and JNK $(\mathrm{P}=0.011$ and $\mathrm{P}=0.009$ respectively), and no significant difference was found in the gene expression rates and protein content of MKK and JNK compared with the control group (Figure 4). These results indicated the combined application of CTX and PP242 in BRAF wild-type Caco-2 cells could effectively inhibit the activation of EGFR and its downstream signaling pathways.

\section{Knockdown or overexpression of EGFR cannot cause changes in the phosphorylation of EGFR downstream signaling pathways in HT-29 cells}

To clarify the reasons for the inconsistent changes in EGFR downstream signaling pathways in HT-29 cells and Caco-2 cells after combination therapy, we knockeddown and overexpressed EGFR in vitro and observed the changes to key molecules in EGFR downstream signaling pathways. In HT-29 cells, the expression, protein content, and phosphorylation rates of EGFR decreased after $E G F R$ knockdown by siRNA (Figure $5 A-5 C$ ), and after overexpressing $E G F R$ by recombinant plasmid, the expression, protein content, and level of phosphorylation of EGFR increased (Figure $5 D-5 F$ ). The data indicated that we successfully interfered with EGFR expression in HT-29 cells in vitro. We then determined the expression rates, protein content, and phosphorylation of key molecules downstream of EGFR after EGFR knockdown and overexpression, and the results showed that regardless of knockdown (Figure $5 A-5 C$ ) or overexpression (Figure 5D-5F) of $E G F R$, there was no specific significant effect on the gene expression, protein content, and phosphorylation rates of MEK, ERK, MKK, and JNK. These results may indicate that, in $B R A F$ mutant HT-29 cells, EGFR activation cannot be successfully transmitted to its downstream signaling pathways.

\section{Knockdown or overexpression of EGFR led to changes in the activation of EGFR downstream signaling pathways in BRAF wild-type Caco-2 cells}

We also knocked down and overexpressed EGFR in $B R A F$ wild-type Caco-2 cells to detect changes in EGFR downstream signaling pathways, and the data confirmed that we successfully knocked down $E G F R$ with siRNA (Figure $6 A-6 C$ ) and overexpressed EGFR with recombinant plasmids (Figure 6D-6F). However, unlike the experimental results in HT-29 cells, after EGFR knockdown, the phosphorylation of MEK, ERK, and the phosphorylation of MKK, JNK were all down-regulated (Figure 6A-6C). After $E G F R$ overexpression, the phosphorylation of MEK, ERK, MKK, and JNK increased (Figure 6D-6F), while no significant changes in gene expression and protein content were observed. Based on the experimental results in the HT-29 and Caco-2 cell lines, we speculate that in BRAF wild-type Caco-2 cells, changes in the activation level of EGFR smoothly transmitted to its downstream channel, which is different from BRAF mutant HT-29 cells. This may explain why the combination application of CTX and PP242 in HT-29 and Caco-2 cell lines caused different changes in the EGFR downstream signaling pathway.

\section{Discussion}

In this study, we further demonstrated the effectiveness of combination therapy by applying CTX and PP242 to CRC cell lines and mouse in vitro xenograft models. We also conducted a preliminary study on changes in the downstream signaling pathways of EGFR after the combined application of CTX and PP242 by detecting the phosphorylation of key molecules in CRC cell lines. We first explored the changes of the signaling pathways that 


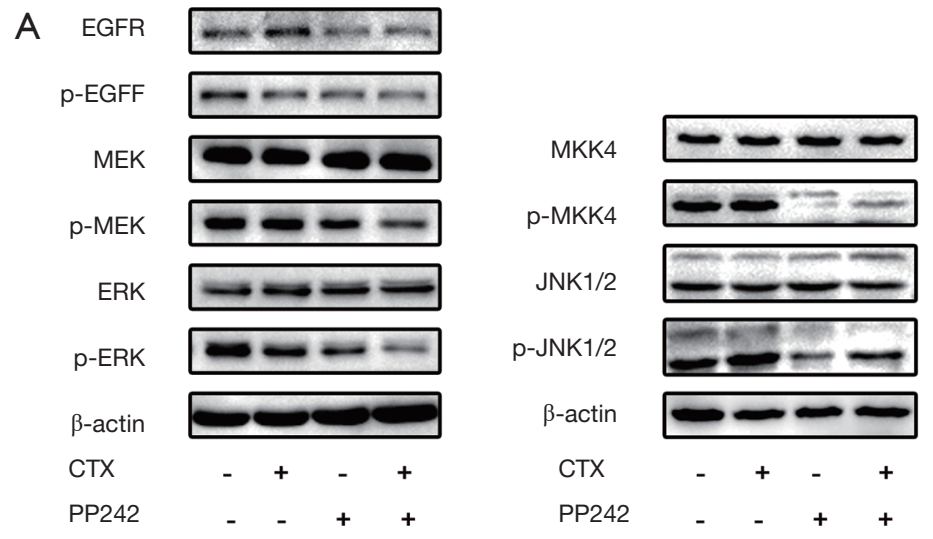

B
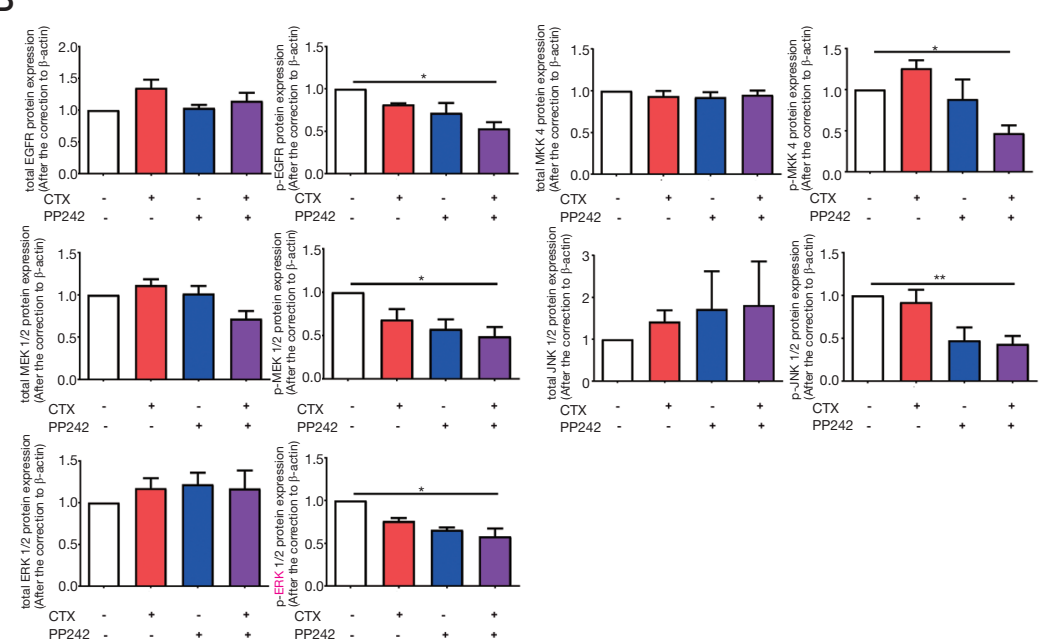

C
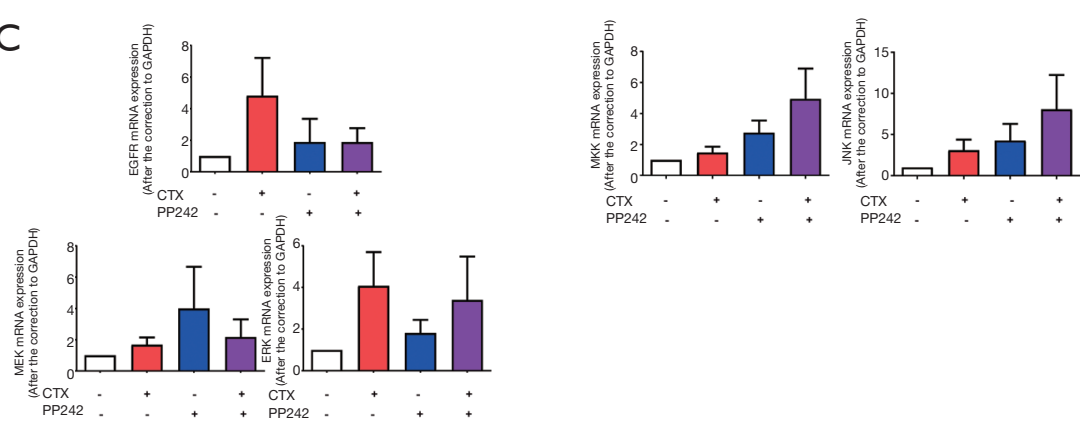

Figure 4 Combined application of CTX and PP242 inhibited the phosphorylation of key molecules of EGFR downstream signaling pathways in Caco-2 cells. Caco-2 cells were untreated or treated with $20 \mu \mathrm{g} / \mathrm{mL}$ CTX and $1 \mu \mathrm{mol} / \mathrm{L} \mathrm{PP2} 22$ alone or in combination for 96 h. (A) The protein content of cellular total and phosphorylated EGFR, MEK, ERK, MKK and JNK contents were determined by western blotting. (B) The semi-quantitative analysis of cellular total and phosphorylated EGFR, MEK, ERK, MKK and JNK contents was performed based on the relative density values of the bands of western blotting. The relative density value of the control group was set to one. (C) qPCR was used to measure the mRNA expression of EGFR, MEK, ERK, MKK and JNK in different treatment groups relative to the control group. The relative mRNA expression of the control group was set to one. Each bar represents the mean \pm SEM. All data were derived from at least three separate experiments. * $\mathrm{P}<0.05$; ** $\mathrm{P}<0.01$. CTX, cetuximab; EGFR, epidermal growth factor receptor; MEK, mitogen-activated protein kinase kinase; ERK, extracellular regulated protein kinase; MKK, MEK 4/7; JNK, c-Jun N-terminal kinase; qPCR, quantitative real time polymerase chain reaction; SEM, standard error of mean. 
A

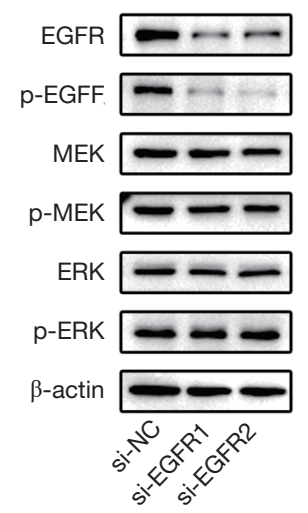

D

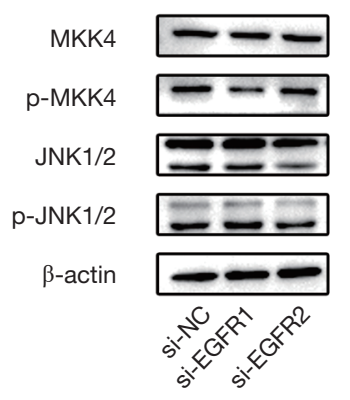

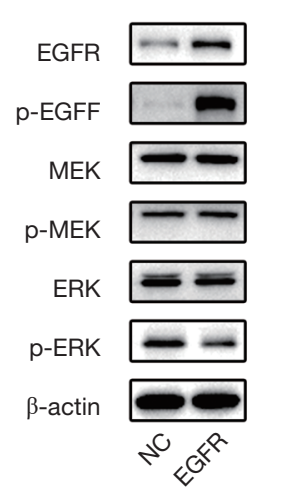

MKK4

p-MKK4 -

JNK1/2

p-JNK1/2 $\square$

$\beta$-actin

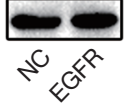

B
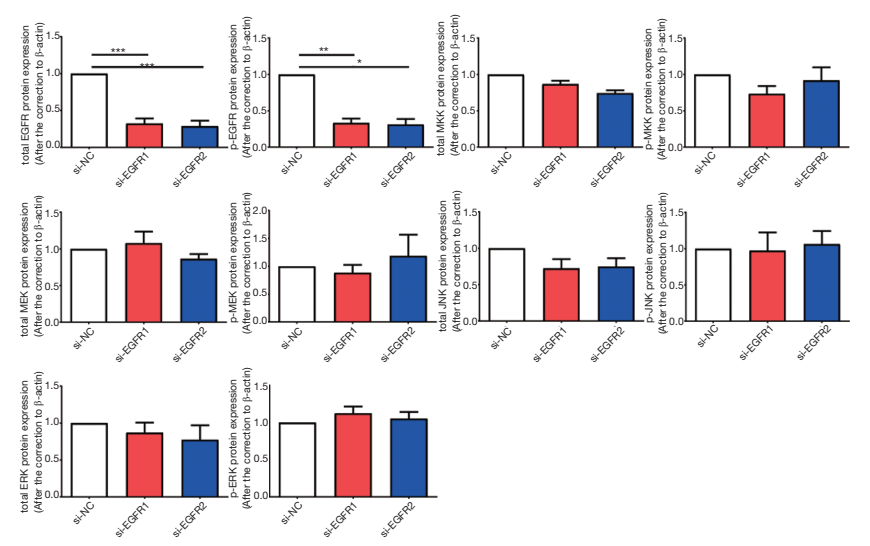

C

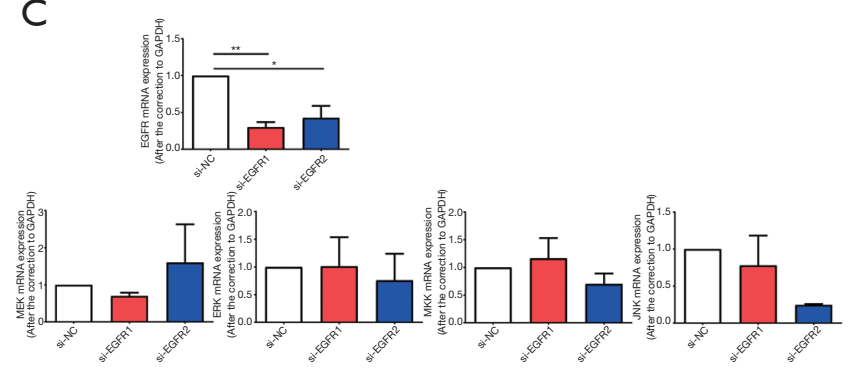

E
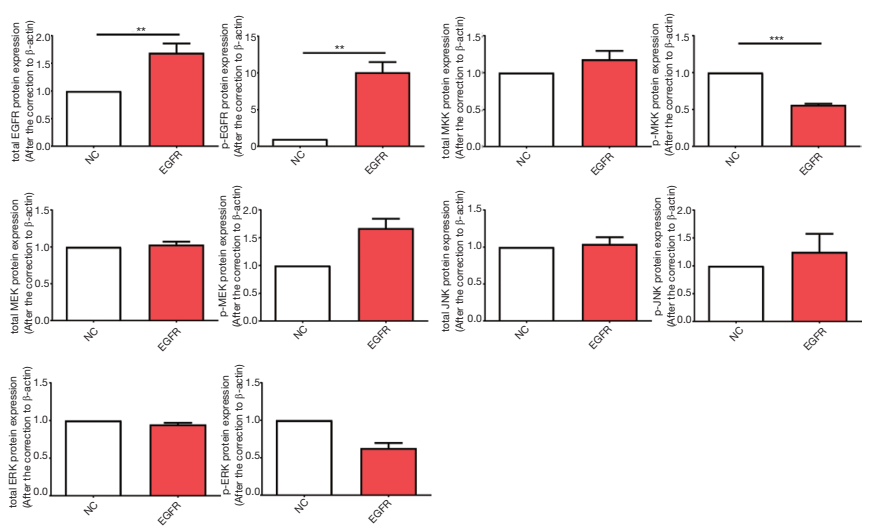

F
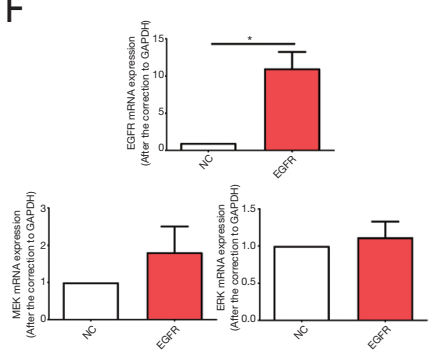

Figure 5 Knockdown or overexpression of EGFR cannot cause changes in the phosphorylation of EGFR downstream signaling pathways in HT-29 cells. In HT-29 cells, siRNA and recombinant plasmids were used to knock down and overexpress EGFR, respectively. (A,D) The protein content of cellular total and phosphorylated EGFR, MEK, ERK, MKK and JNK contents were determined by western blotting after knockdown (A) and overexpression of EGFR (D). (B,E) The semi-quantitative analysis of cellular total and phosphorylated EGFR, MEK, ERK, MKK and JNK contents was performed based on the relative density values of the bands of western blotting. The relative density value of the control group was set to one. (C,F) qPCR was used to measure the mRNA expression of EGFR, MEK, ERK, MKK and JNK in EGFR knockdown groups (C) and EGFR overexpression group (F) relative to the control group. The relative mRNA expression of the control group was set to one. Each bar represents the mean \pm SEM. All data were derived from at least three separate experiments. *, $\mathrm{P}<0.05$; **, $\mathrm{P}<0.01$; ***, $\mathrm{P}<0.001$. EGFR, epidermal growth factor receptor; siRNA, small interfering RNA; MEK, mitogen-activated protein kinase kinase; ERK, extracellular regulated protein kinase; MKK, MEK 4/7; JNK, c-Jun N-terminal kinase; qPCR, quantitative real time polymerase chain reaction; SEM, standard error of mean. 
A

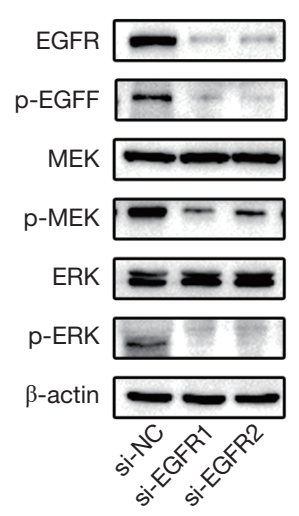

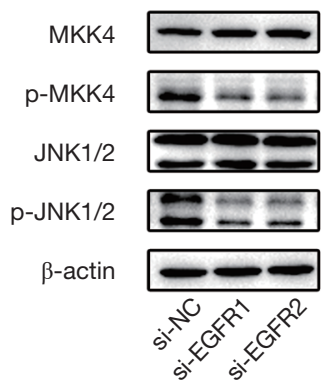

D

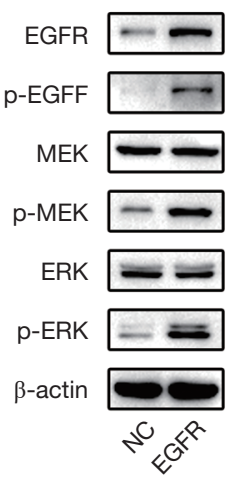

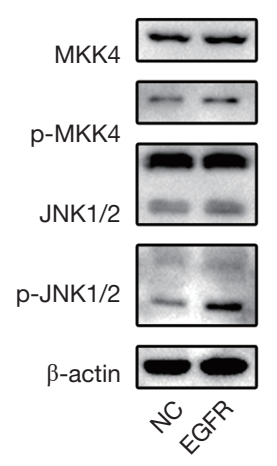

B
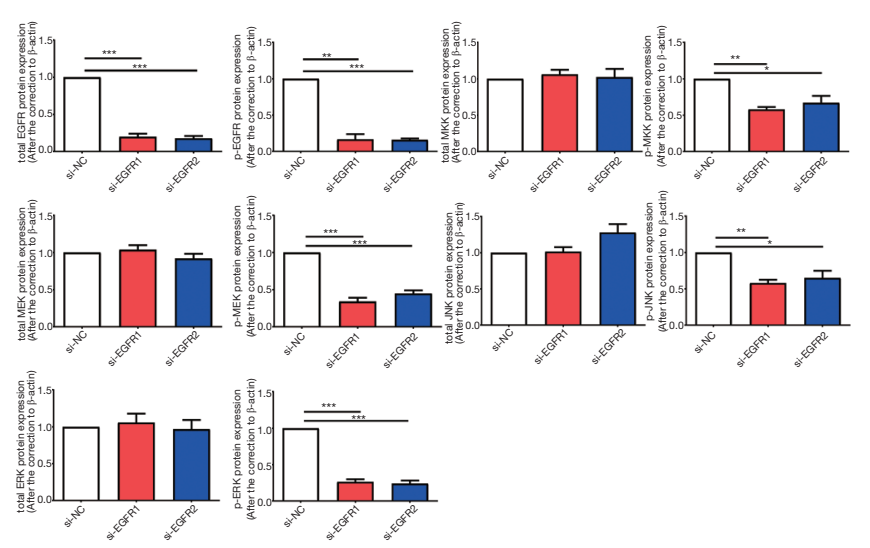

C

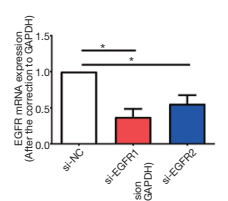

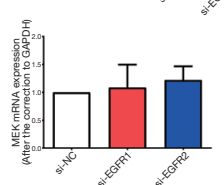
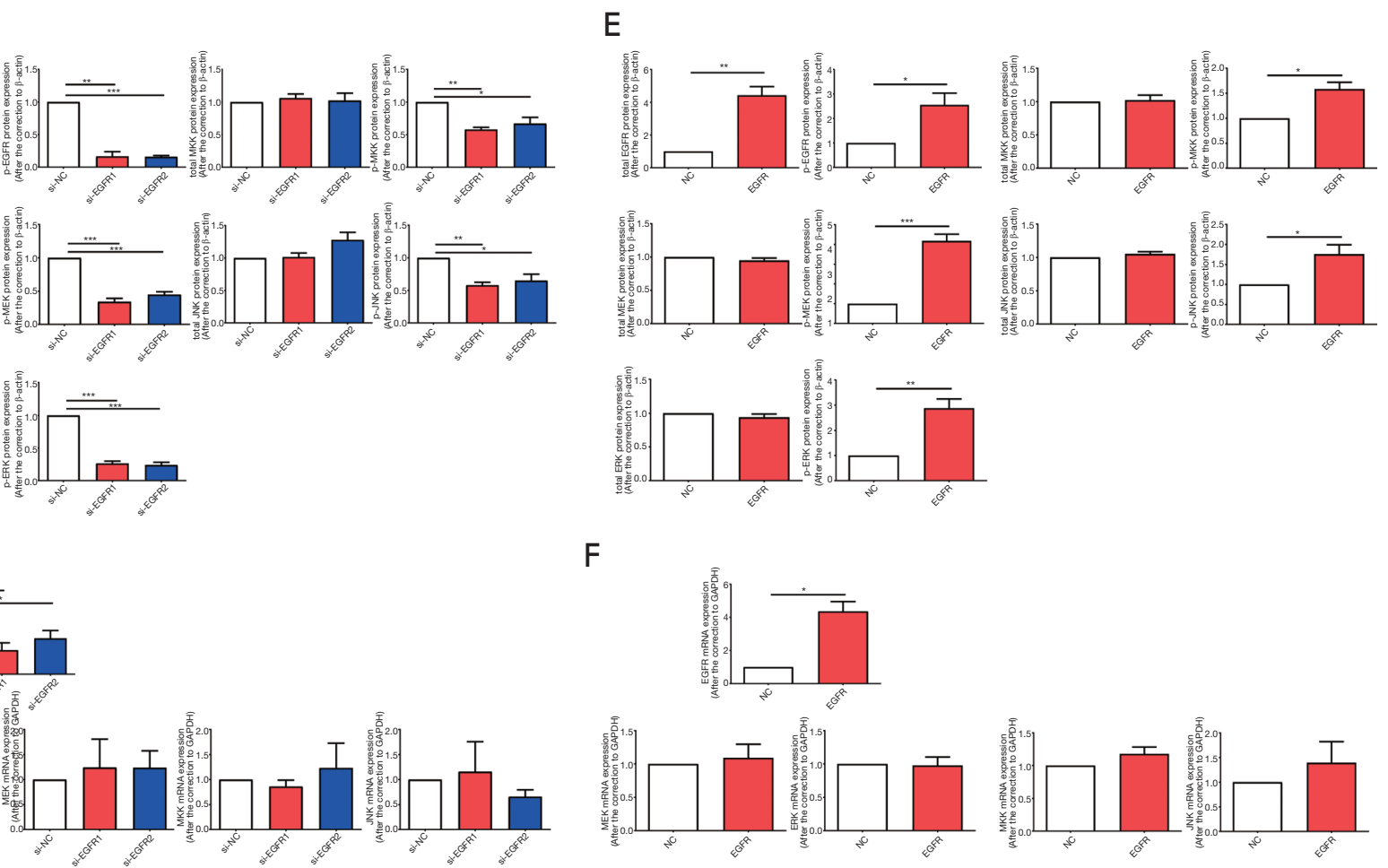

$\mathrm{F}$
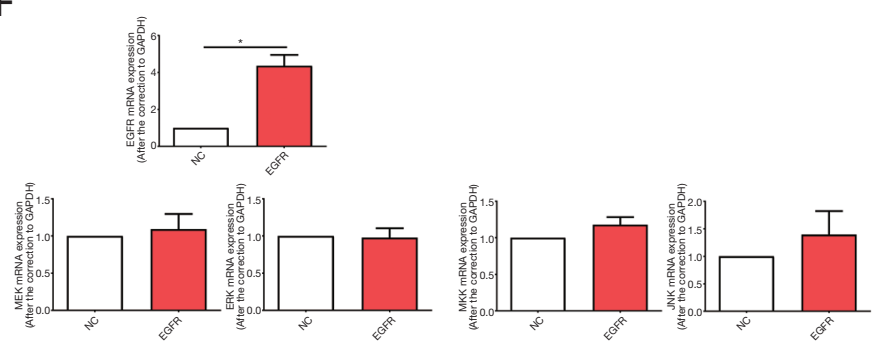

Figure 6 Knockdown or overexpression of EGFR can lead to changes in the activation rates of EGFR downstream signaling pathways in Caco-2 cells. In Caco-2 cells, siRNA and recombinant plasmids were used to knock down and overexpress EGFR, respectively. (A,D) The protein content of cellular total and phosphorylated EGFR, MEK, ERK, MKK and JNK contents were determined by western blotting after knockdown (A) and overexpression of EGFR (D). (B,E) The semi-quantitative analysis of cellular total and phosphorylated EGFR, MEK, ERK, MKK and JNK contents was performed based on the relative density values of the bands of western blotting. The relative density value of the control group was set to one. (C,F) qPCR was used to measure the mRNA expression of EGFR, MEK, ERK, MKK and JNK in EGFR knockdown groups (C) and EGFR overexpression group (F) relative to the control group. The relative mRNA expression of the control group was set to one. Each bar represents the mean \pm SEM. All data were derived from at least three separate experiments. *, $\mathrm{P}<0.05 ;{ }^{* *}, \mathrm{P}<0.01$; ${ }^{* * *}, \mathrm{P}<0.001$. EGFR, epidermal growth factor receptor; siRNA, small interfering RNA; MEK, mitogen-activated protein kinase kinase; ERK, extracellular regulated protein kinase; MKK, MEK 4/7; JNK, c-Jun N-terminal kinase; qPCR, quantitative real time polymerase chain reaction; SEM, standard error of mean. 
may occur in CRC cells after the combined application of CTX and PP242, which may provide further theoretical evidence for combined targeted therapy to move toward clinical applications.

In the HT-29 cell line, we found that CTX monotherapy could neither reduce the proliferation of tumor cells, nor inhibit the cell cycle and induce apoptosis, and in the detection of molecule phosphorylation rates, we found that knockdown or overexpression of EGFR in HT-29 cells did not cause significant changes in the downstream molecule phosphorylation rates of EGFR. In a study on gold nanoparticles, CTX-loaded nanoparticles did not significantly cause apoptosis in HT-29 cells compared to unloaded nanoparticles (36), and in HT-29 cells, the application of anti-EGFR antibody alone could not change the tumor cell spheroidization size or proliferation rate (37). These results are consistent with our research. The HT29 cell line contains the $B R A F$ V600E mutation, which may be the cause of the inconsistency of cell signal transduction in the HT-29 cell line and Caco-2 cell line. We speculate that due to the existence of BRAF mutation in the HT-29 cells, the changes of EGFR phosphorylation are unable to transmit to the downstream MEK-ERK signaling pathway through BRAF, which greatly reduces the effect of antiEGFR therapy on HT-29 cells. In clinical practice, CRC patients with $B R A F$ V600E mutations will not benefit from anti-EGFR therapy $(11,38)$. Therefore, in $B R A F$-mutated CRC patients, it is necessary to add inhibitory therapies to other targets such as mTOR inhibitors on the basis of anti-EGFR therapy. In our experiments, the combined application of CTX and PP242 had a significant anti-tumor effect on $B R A F$-mutated HT-29 cells, suggesting that dualtargeted therapy for EGFR and mTOR may improve the efficacy of anti-EGFR therapy resistant patients.

In experiments with HT-29 cells, we observed a significant up-regulation of EGFR phosphorylation after using PP242, accompanied by significant up-regulation of downstream MEK and ERK phosphorylation. A study has shown that in mouse keratinocytes, the pharmacological inhibition of mTORC1 could inhibit the protein expression and activity of EGFR and ErbB2, suggesting the pharmacological inhibition of mTORC1 inhibits EGF-induced EGFR degradation by inhibiting lysosomal biogenesis and activity (39). Another study has pointed out that the upregulated EGFR may be the reason for the resistance to mTOR inhibitors in CRC cells, as the combined inhibition of ErbB and the PI3K/ mTOR signaling pathway inhibits the proliferation activity of CRC cells (40). This may explain why some CRC cell lines are resistant to mTOR inhibitors. However, we have not observed this phenomenon in Caco- 2 cells. In Caco2 cells, the application of PP242 did not up-regulate EGFR gene expression and phosphorylation, but instead led to down-regulation of EGFR downstream MEK and ERK phosphorylation. We speculate this may be related to different genetic states in the two cell lines. In $B R A F-$ mutant HT-29 cells, the inhibitory effect of PP242 on mTOR will be negatively fed back to the upstream EGFR and its downstream MEK-ERK signaling pathway, resulting in increased phosphorylation of EGFR, MEK, and ERK (41). After applying CTX on the basis of PP242, the phosphorylation of EGFR was significantly lower than that of PP242 monotherapy, which is another important reason why we tried to combine PP242 and CTX. Research by Corcoran et. al also showed that in $B R A F$-mutated CRCs, inhibition of the downstream signaling pathway of EGFR led to negative feedback up-regulation of EGFR phosphorylation, and combined inhibition of EGFR and downstream signaling pathways could improve efficacy in vitro and in vivo (42). Although the reduction of EGFR phosphorylation cannot be smoothly transmitted to its downstream MEK-ERK signaling pathway due to the presence of $B R A F$ mutations, the reduction may also affect cell proliferation, differentiation, and apoptosis through other signaling pathways. In $B R A F$ wild-type Caco-2 cells, studies have shown that the expression of plateletderived growth factor receptor alpha (PDGFRA) is significantly higher than that of other CRC cell lines (43), suggesting that PDGFRA may also be a possible target for combination therapy. However, these findings require further investigation in subsequent experiments.

Although anti-EGFR monoclonal antibodies such as CTX and panitumumab have been widely used in the treatment of mCRC, the activity of anti-EGFR monotherapy is still limited to a subset of patients with mCRC. It may be because some patients have gene mutations such as KRAS and BRAF that interfere with the normal transmission of the EGFR signaling pathway (11). Long-term use of anti-EGFR therapy may also lead to drug resistance in some patients (12). In addition, autophagy is also believed to be related to resistance to anti-EGFR therapy. A study has shown that CTX reduce the expression of miR-216b and up-regulate Beclin-1 to increase autophagy in CRC cells, thereby antagonizing CTX-induced cell death (44). Therefore, the co-targeting of EGFR and autophagy signals may become an emerging treatment 
strategy for mCRC. A study showed that for microsatellite instability-high (MSI-H) CRC cells, autophagy inhibitors combined with EGFR monoclonal antibody and checkpoint inhibitors is an effective choice (45). In addition, autophagy inhibitors such as chloroquine and hydroxychloroquine have also been tried to reverse the resistance of anti-EGFR therapy in preclinical studies and early clinical trials (46). This reveals another possibility of reversing resistance to anti-EGFR therapy.

Research on the combined inhibition of EGFR and mTOR in the treatment of CRC dates to 2006. Researchers combined the mTOR inhibitor rapamycin with the EGFR inhibitor erlotinib in a variety of cell lines including CRC cell lines, and observed a combined inhibitory effect in some (47). A later study showed that the combined application of erlotinib and rapamycin inhibited the growth of KRAS wild-type or mutant CRC cell lines compared to erlotinib monotherapy (48). As research progressed, more mTOR inhibitors were applied in investigations on CRC treatment, and the combination of CTX and the mTOR inhibitor everolimus was found to reduce tumor size in patient-derived tumor cells xenograft models and inhibit the Akt and ERK signaling pathways (49). Clinical trials have also proved the effectiveness of EGFR and mTOR dual-targeted therapy for mCRC. A phase I clinical study combining CTX, irinotecan and the mTOR inhibitor everolimus in the second-line treatment of mCRC showed that when administered at a specific dose, the progressionfree survival (PFS) was 5.9 months, and the overall survival (OS) was 17.5 months (50). Another phase IB/II clinical study in combination with panitumumab, irinotecan and everolimus, a second-line treatment of KRAS wild-type mCRC, showed that the median PFS was 5.6 months and the median OS was 10.8 months. Its toxicity is expected and controllable (51). The combination of dual inhibitors of mTORC1 and mTORC2 and anti-EGFR therapy has also been investigated in the treatment of CRC. One study showed the combined application of the EGFR inhibitor GC118 and the dual mTOR inhibitor BEZ-235 reduced the size of tumors in mice in vivo (52). In another study, PP242 was seen to enhance the phosphorylation of EGFR in CRC cell lines, and the combined application of PP242 and erlotinib induced apoptosis of CRC cells and reduced the volume of mouse xenografts in vivo (53), which further confirms our experimental results. Our study explored the changes in the MEK-ERK and the MKK-JNK signaling pathway in the EGFR downstream of CRC cells under the combined action of CTX and PP242, but the changes in other signaling pathways and the changes of CRC cells under different gene states still needs to be clarified. The driver genes closely related to the progression and metastasis of CRC, such as KRAS, APC, TP53 and SMAD4 (54), whose impact of their mutations on the dual-targeted therapy of EGFR and mTOR also need to be further studied. The lack of theoretical basis has brought challenges to the clinical development of mTOR and EGFR dualtargeted therapy. Therefore, in future studies, we still need to further clarify the changes caused by the combined inhibition of EGFR and mTOR on various signaling pathways downstream of EGFR, and the impact of different genotypes of CRC on the sensitivity of this scheme.

To conclude, our research further demonstrated the effectiveness of the combined application of CTX and PP242 in inhibiting the proliferation of CRC cell lines and the tumor growth of mouse xenografts. We revealed that in the $B R A F$ wild-type Caco-2 cell line, the combined application of CTX and PP242 inhibited the proliferation, differentiation, and apoptosis of CRC cells by inhibiting the activation of the EGFR downstream MEK-ERK and MKKJNK signaling pathways. In addition, we found that in BRAF mutant HT-29 cells, PP242 monotherapy resulted in negative feedback up-regulation of EGFR phosphorylation, accompanied by significant up-regulation of downstream MEK and ERK phosphorylation. Our research further explored the molecular mechanism of combined inhibition of EGFR and mTOR in the treatment of CRC, and provides more theoretical evidence for clinical applications in the future.

\section{Acknowledgments}

Funding: This work was supported by the Nanjing Health and Family Planning Commission key program (ZKX17012) and Jiangsu Health and Family Planning Commission development program (H2017042).

\section{Footnote}

Reporting Checklist: The authors have completed the ARRIVE reporting checklist. Available at https://dx.doi. org/10.21037/jgo-21-467

Data Sharing Statement: Available at https://dx.doi. org/10.21037/jgo-21-467 
Conflicts of Interest: All authors have completed the ICMJE uniform disclosure form (available at https://dx.doi. org/10.21037/jgo-21-467). The authors have no conflicts of interest to declare.

Etbical Statement: The authors are accountable for all aspects of the work in ensuring that questions related to the accuracy or integrity of any part of the work are appropriately investigated and resolved. Experiments were performed under a project license (No.: 2019AE01054) granted by the Nanjing Drum Tower Hospital ethics committee, in compliance with China national or institutional guidelines for the care and use of animals.

Open Access Statement: This is an Open Access article distributed in accordance with the Creative Commons Attribution-NonCommercial-NoDerivs 4.0 International License (CC BY-NC-ND 4.0), which permits the noncommercial replication and distribution of the article with the strict proviso that no changes or edits are made and the original work is properly cited (including links to both the formal publication through the relevant DOI and the license). See: https://creativecommons.org/licenses/by-nc-nd/4.0/.

\section{References}

1. Gu M, Thapa S. Colorectal cancer in the United States and a review of its heterogeneity among Asian American subgroups. Asia Pac J Clin Oncol 2020;16:193-200.

2. Fu J, Zhu J, Du F, et al. Prognostic inflammatory index based on preoperative peripheral blood for predicting the prognosis of colorectal cancer patients. Cancers (Basel) 2020;13:3.

3. Ahmed M. Colon cancer: a clinician's perspective in 2019. Gastroenterology Res 2020;13:1-10.

4. Parmar A, Chan KKW, Ko YJ. Metastatic colorectal cancer: therapeutic options for treating refractory disease. Curr Oncol 2019;26:S24-32.

5. Willems E, Gerne L, George C, et al. Adverse effects of bevacizumab in metastatic colorectal cancer: a case report and literature review. Acta Gastroenterol Belg 2019;82:322-5.

6. Chen Z, Jiang L. The clinical application of fruquintinib on colorectal cancer. Expert Rev Clin Pharmacol 2019;12:713-21.

7. Kreidieh M, Mukherji D, Temraz S, et al. Expanding the scope of immunotherapy in colorectal cancer: current clinical approaches and future directions. Biomed Res Int
2020;2020:9037217.

8. Martinelli E, Ciardiello D, Martini G, et al. Implementing anti-epidermal growth factor receptor (EGFR) therapy in metastatic colorectal cancer: challenges and future perspectives. Ann Oncol 2020;31:30-40.

9. Wang C, Ouyang C, Cho M, et al. Wild-type APC is associated with poor survival in metastatic microsatellite stable colorectal cancer. Oncologist 2021;26:208-14.

10. Prahallad A, Sun C, Huang S, et al. Unresponsiveness of colon cancer to BRAF(V600E) inhibition through feedback activation of EGFR. Nature 2012;483:100-3.

11. Loree JM, Dowers A, Tu D, et al. Expanded low allele frequency RAS and BRAF V600E testing in metastatic colorectal cancer as predictive biomarkers for cetuximab in the randomized CO.17 trial. Clin Cancer Res 2021;27:52-9.

12. Lorenzato A, Magrì A, Matafora V, et al. Vitamin C restricts the emergence of acquired resistance to EGFRtargeted therapies in colorectal cancer. Cancers (Basel) 2020;12:685.

13. Shahjehan F, Kamatham S, Chandrasekharan C, et al. Binimetinib, encorafenib and cetuximab (BEACON Trial) combination therapy for patients with BRAF V600Emutant metastatic colorectal cancer. Drugs Today (Barc) 2019;55:683-93.

14. Miele E, Abballe L, Spinelli GP, et al. BRAF mutant colorectal cancer: ErbB2 expression levels as predictive factor for the response to combined BRAF/ErbB inhibitors. BMC Cancer 2020;20:129.

15. Zhou S, Xu H, Tang Q, et al. Dipyridamole enhances the cytotoxicities of trametinib against colon cancer cells through combined targeting of HMGCS1 and MEK pathway. Mol Cancer Ther 2020;19:135-46.

16. Zhang P, Li R, Xiao H, et al. BRD4 inhibitor AZD5153 suppresses the proliferation of colorectal cancer cells and sensitizes the anticancer effect of PARP inhibitor. Int J Biol Sci 2019;15:1942-54.

17. Khan K, Valeri N, Dearman C, et al. Targeting EGFR pathway in metastatic colorectal cancer- tumour heterogeniety and convergent evolution. Crit Rev Oncol Hematol 2019;143:153-63.

18. Panda M, Biswal BK. Cell signaling and cancer: a mechanistic insight into drug resistance. Mol Biol Rep 2019;46:5645-59.

19. Zhao HF, Wang J, Tony To SS. The phosphatidylinositol 3-kinase/Akt and c-Jun N-terminal kinase signaling in cancer: alliance or contradiction? (Review). Int J Oncol 2015;47:429-36. 
20. Leite FGG, Torres AA, De Oliveira LC, et al. c-Jun integrates signals from both MEK/ERK and MKK/ JNK pathways upon vaccinia virus infection. Arch Virol 2017;162:2971-81.

21. Sugiyama E, Togashi Y, Takeuchi Y, et al. Blockade of EGFR improves responsiveness to PD-1 blockade in EGFR-mutated non-small cell lung cancer. Sci Immunol 2020;5:eaav3937.

22. Capalbo C, Belardinilli F, Filetti M, et al. Effective treatment of a platinum-resistant cutaneous squamous cell carcinoma case by EGFR pathway inhibition. Mol Clin Oncol 2018;9:30-4.

23. Mauri G, Bonazzina E, Amatu A, et al. The evolutionary landscape of treatment for BRAFV600E mutant metastatic colorectal cancer. Cancers (Basel) 2021;13:137.

24. Mirza-Aghazadeh-Attari M, Ekrami EM, Aghdas SAM, et al. Targeting PI3K/Akt/mTOR signaling pathway by polyphenols: Implication for cancer therapy. Life Sci 2020;255:117481.

25. Heras-Sandoval D, Pérez-Rojas JM, Pedraza-Chaverri J. Novel compounds for the modulation of mTOR and autophagy to treat neurodegenerative diseases. Cell Signal 2020;65:109442.

26. Krencz I, Sebestyen A, Khoor A. mTOR in lung neoplasms. Pathol Oncol Res 2020;26:35-48.

27. Srivastava RK, Li C, Khan J, et al. Combined mTORC1/ mTORC2 inhibition blocks growth and induces catastrophic macropinocytosis in cancer cells. Proc Natl Acad Sci U S A 2019;116:24583-92.

28. Kawata T, Tada K, Kobayashi M, et al. Dual inhibition of the mTORC1 and mTORC2 signaling pathways is a promising therapeutic target for adult T-cell leukemia. Cancer Sci 2018;109:103-11.

29. Musa F, Alard A, David-West G, et al. Dual mTORC1/2 inhibition as a novel strategy for the resensitization and treatment of platinum-resistant ovarian cancer. Mol Cancer Ther 2016;15:1557-67.

30. Marquard FE, Jücker M. PI3K/AKT/mTOR signaling as a molecular target in head and neck cancer. Biochem Pharmacol 2020;172:113729.

31. Cheng L, Xia Z, Bian X, et al. Combination of cetuximab and PP242 synergistically suppress the progression of wild-type KRAS colorectal carcinoma. Onco Targets Ther 2015;8:3185-92.

32. Tanaka K, Sasayama T, Irino Y, et al. Compensatory glutamine metabolism promotes glioblastoma resistance to $\mathrm{mTOR}$ inhibitor treatment. J Clin Invest 2015;125:1591-602.
33. Sun X, Kaufman PD. Ki-67: more than a proliferation marker. Chromosoma 2018;127:175-86.

34. Piera-Velazquez S, Jimenez SA. Endothelial to mesenchymal transition: role in physiology and in the pathogenesis of human diseases. Physiol Rev 2019;99:1281-324.

35. Boice A, Bouchier-Hayes L. Targeting apoptotic caspases in cancer. Biochim Biophys Acta Mol Cell Res 2020;1867:118688.

36. Liszbinski RB, Romagnoli GG, Gorgulho CM, et al. Anti-EGFR-coated gold nanoparticles in vitro carry 5-fluorouracil to colorectal cancer cells. Materials (Basel) 2020;13:375.

37. Olejniczak-K der A, Szary ska M, Wro ska A, et al. Effects of 5-FU and anti-EGFR antibody in combination with ASA on the spherical culture system of HCT116 and HT29 colorectal cancer cell lines. Int J Oncol 2019;55:223-42.

38. Ducreux M, Chamseddine A, Laurent-Puig P, et al. Molecular targeted therapy of BRAF-mutant colorectal cancer. Ther Adv Med Oncol 2019;11:1758835919856494.

39. Asrani K, Murali S, Lam B, et al. mTORC1 feedback to AKT modulates lysosomal biogenesis through MiT/TFE regulation. J Clin Invest 2019;129:5584-99.

40. Belmont PJ, Jiang P, McKee TD, et al. Resistance to dual blockade of the kinases PI3K and mTOR in KRAS-mutant colorectal cancer models results in combined sensitivity to inhibition of the receptor tyrosine kinase EGFR. Sci Signal 2014; 7:ra107.

41. Hoang B, Benavides A, Shi Y, et al. The PP242 mammalian target of rapamycin (mTOR) inhibitor activates extracellular signal-regulated kinase (ERK) in multiple myeloma cells via a target of rapamycin complex 1 (TORC1)/eukaryotic translation initiation factor 4E (eIF-4E)/RAF pathway and activation is a mechanism of resistance. J Biol Chem 2012;287:21796-805.

42. Corcoran RB, Ebi H, Turke AB, et al. EGFR-mediated reactivation of MAPK signaling contributes to insensitivity of BRAF mutant colorectal cancers to RAF inhibition with vemurafenib. Cancer Discov 2012;2:227-35.

43. Medico E, Russo M, Picco G, et al. The molecular landscape of colorectal cancer cell lines unveils clinically actionable kinase targets. Nat Commun 2015;6:7002.

44. Chen Z, Gao S, Wang D, et al. Colorectal cancer cells are resistant to anti-EGFR monoclonal antibody through adapted autophagy. Am J Transl Res 2016;8:1190-6.

45. Koustas E, Papavassiliou AG, Karamouzis MV. The role of autophagy in the treatment of BRAF mutant colorectal 
carcinomas differs based on microsatellite instability status. PLoS One 2018;13:e0207227.

46. Koustas E, Karamouzis MV, Mihailidou C, et al. Cotargeting of EGFR and autophagy signaling is an emerging treatment strategy in metastatic colorectal cancer. Cancer Lett 2017;396:94-102.

47. Buck E, Eyzaguirre A, Brown E, et al. Rapamycin synergizes with the epidermal growth factor receptor inhibitor erlotinib in non-small-cell lung, pancreatic, colon, and breast tumors. Mol Cancer Ther 2006;5:2676-84.

48. Li B, Gao S, Wei F, et al. Simultaneous targeting of EGFR and mTOR inhibits the growth of colorectal carcinoma cells. Oncol Rep 2012;28:15-20.

49. Kim JS, Kim JE, Kim K, et al. The impact of cetuximab plus AKT- or mTOR-inhibitor in a patient-derived colon cancer cell model with wild-type RAS and PIK3CA mutation. J Cancer 2017;8:2713-9.

50. Hecht JR, Reid TR, Garrett CR, et al. Phase I study of everolimus, cetuximab and irinotecan as second-line therapy in metastatic colorectal cancer. Anticancer Res 2015;35:1567-73.

51. Townsend A, Tebbutt N, Karapetis C, et al. Phase IB/ II study of second-line therapy with panitumumab, irinotecan, and everolimus (PIE) in KRAS wildtype metastatic colorectal cancer. Clin Cancer Res 2018;24:3838-44.

52. Lee HW, Son E, Lee K, et al. Promising therapeutic efficacy of GC1118, an anti-EGFR antibody, against KRAS mutation-driven colorectal cancer patient-derived xenografts. Int J Mol Sci 2019;20:5894.

53. Wang Q, Wei F, Li C, et al. Combination of mTOR and EGFR kinase inhibitors blocks mTORC1 and mTORC2 kinase activity and suppresses the progression of colorectal carcinoma. PLoS One 2013;8:e73175.

54. Raskov H, Søby JH, Troelsen J, et al. Driver gene mutations and epigenetics in colorectal cancer. Ann Surg 2020;271:75-85.

(English Language Editor: B. Draper)
Cite this article as: Kong L, Zhang Q, Mao J, Cheng L, Shi X, Yu L, Hu J, Yang M, Li L, Liu B, Qian X. A dual-targeted molecular therapy of PP242 and cetuximab plays an antitumor effect through EGFR downstream signaling pathways in colorectal cancer. J Gastrointest Oncol 2021;12(4):1625-1642. doi: 10.21037/jgo-21-467 
A

HT-29 Cells

Ki-67

CD31

Caspase-3

Cleaved caspase-3

$\beta$-actin

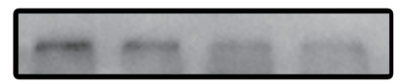

$\mathrm{Ki}-67$

CD31

Caspase-3

Cleaved caspase-3

$\beta$-actin

CTX

PP242
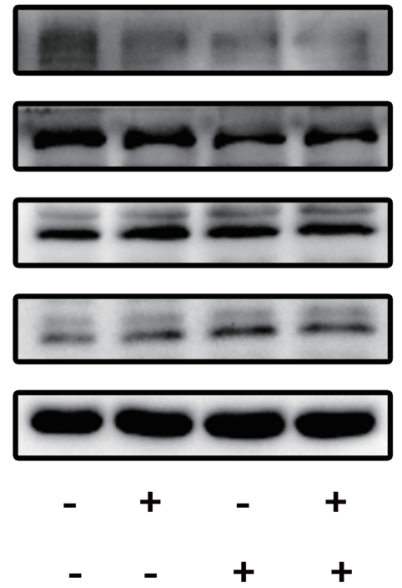
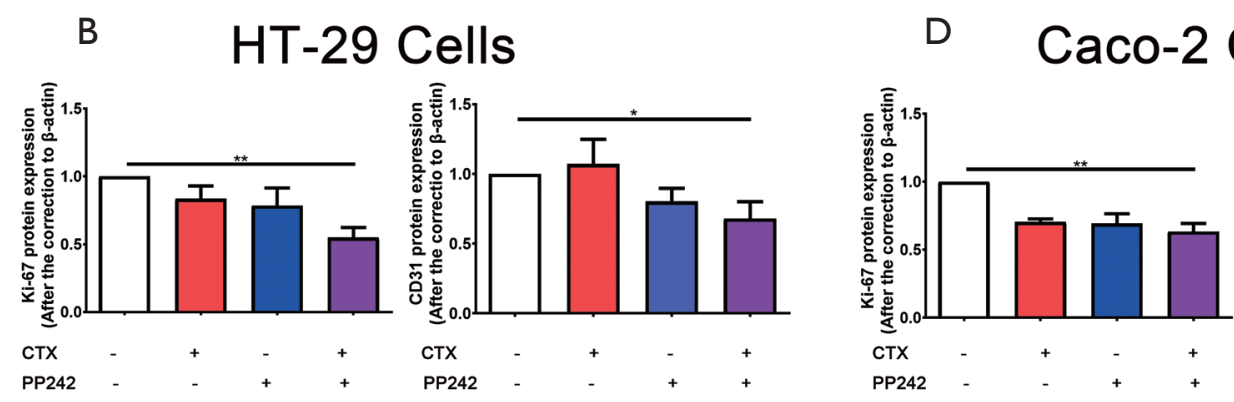

Cells
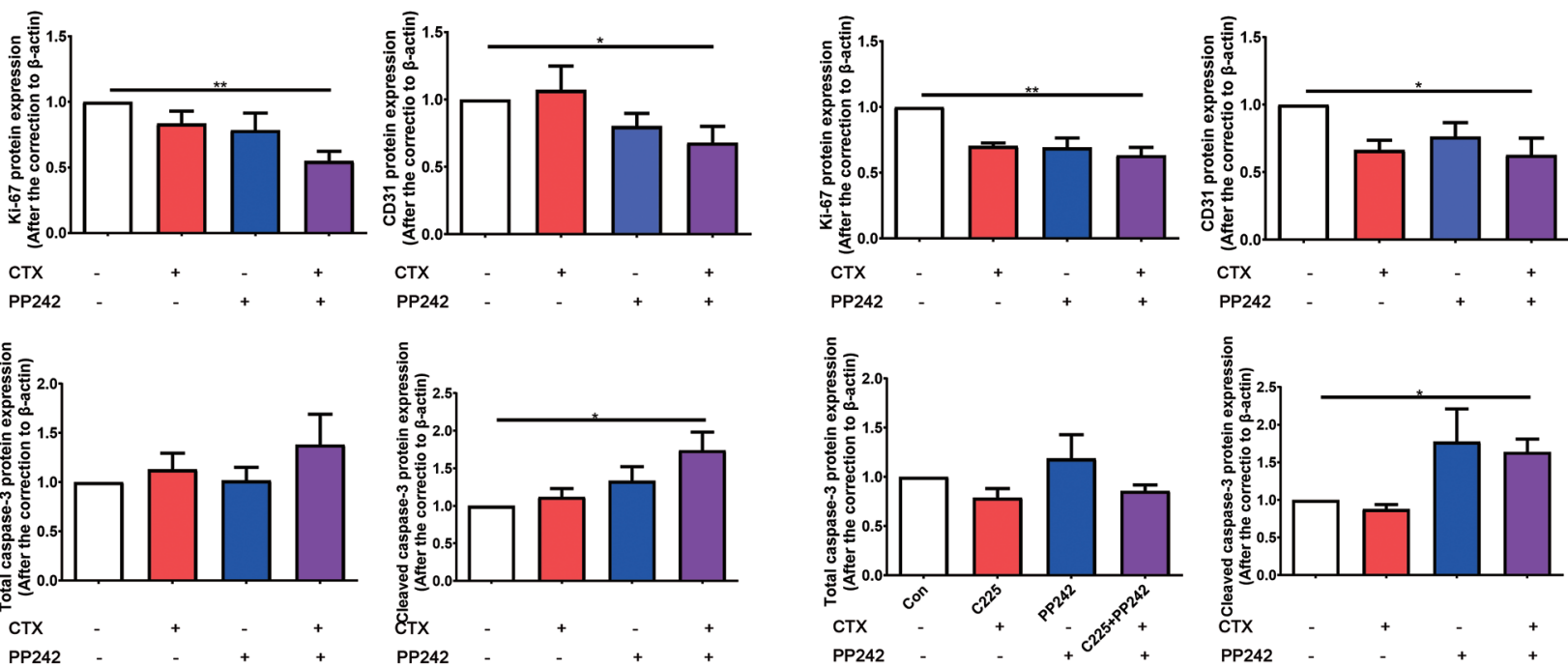

Figure S1 Combined application of CTX and PP242 reduces proliferation-related markers expression and increases apoptosis-related markers expression in CRC cells. HT-29 cells and Caco-2 cells were untreated or treated with $20 \mu \mathrm{g} / \mathrm{mL}$ CTX and $1 \mu \mathrm{mol} / \mathrm{L}$ PP242 alone or in combination for $96 \mathrm{~h}$. (A,C) The protein content of celluar Ki-67, platelet endothelial cell adhesion molecule-1 (CD31), total and cleaved caspase-3 contents were determined by western blotting. (B,D) The semi-quantitative analysis of cellular Ki-67, CD31, total and cleaved caspase-3 contents was performed based on the relative density values of the bands of western blotting. The relative density value of the control group was set to one. Each bar represents the mean \pm SEM. All data were derived from at least three separate experiments. *, $\mathrm{P}<0.05$; **, $\mathrm{P}<0.01$. CTX, cetuximab; CRC, colorectal cancer. SEM, standard error of mean. 


\section{A \\ Ki-67 \\ CD31 \\ Cleaved Caspase-3}

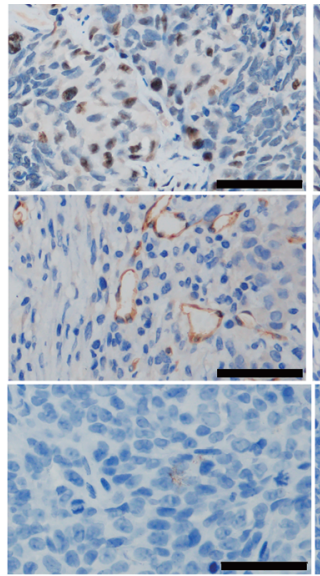

\section{CTX}

PP242

B

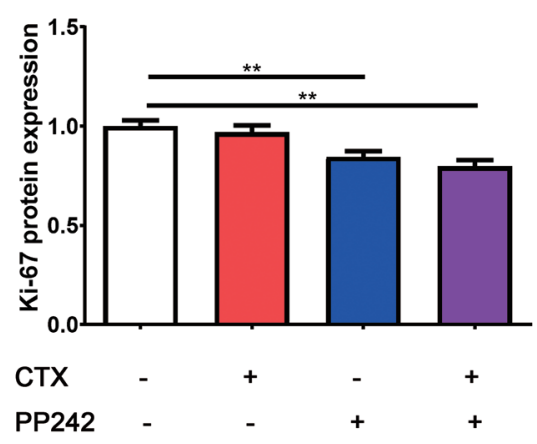

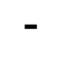

-

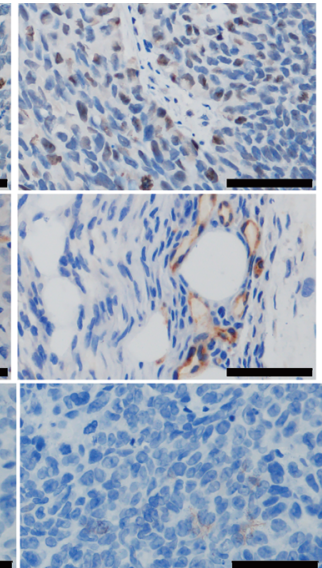

$+$

-

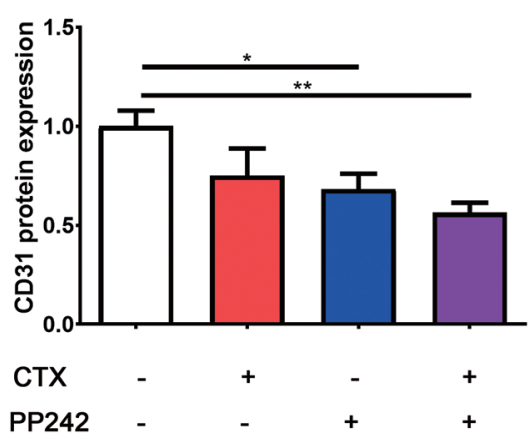

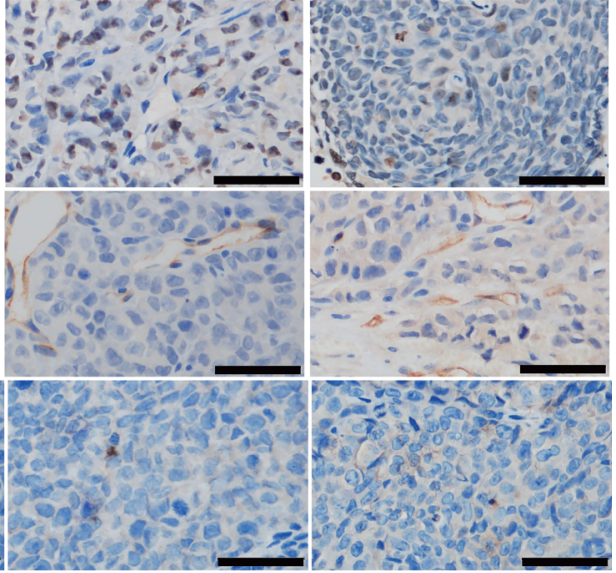

$+$

$+$

Figure S2 Combined application of CTX and PP242 reduces proliferation-related markers expression and increases apoptosis-related markers expression in mouse xenograft tumors. Six-week-old male BALB/c nude mice bearing HT-29 cell xenograft tumors were untreated or treated with $20 \mathrm{mg} / \mathrm{kg}$ CTX and $30 \mathrm{mg} / \mathrm{kg}$ PP242 alone or in combination twice a week. After 2 weeks of treatment, the xenograft tumor was removed and subjected to immunohistochemical analysis. (A) Microscopic view of Ki-67, CD31, and cleaved caspase-3 immunohistochemistry. (B) The semi-quantitative analysis of Ki-67, CD31, and cleaved caspase-3 immunohistochemistry. All data were derived from at least three separate fields of view under the microscope. The relative density value of the control group was set to 1 . Each bar represents the mean \pm SEM. Scale bar: $50 \mu \mathrm{m} .{ }^{*}, \mathrm{P}<0.05 ;{ }^{* *}, \mathrm{P}<0.01$; ${ }^{* * *}, \mathrm{P}<0.001$. CTX, cetuximab. SEM, standard error of mean. 
A

HT-29 Cells

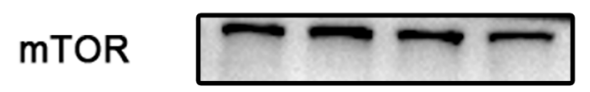

p-mTOR

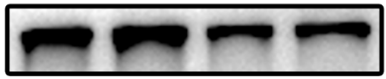

$\beta$-actin

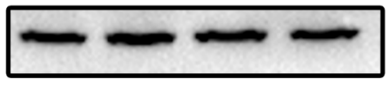

CTX

PP242

C
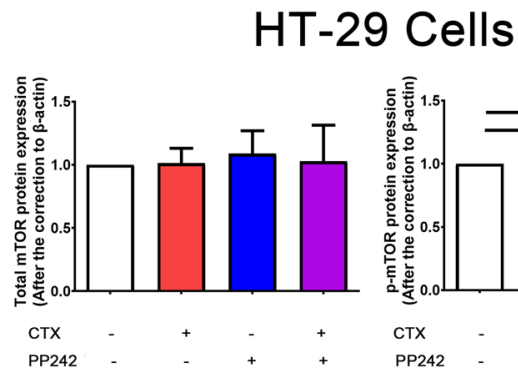

B

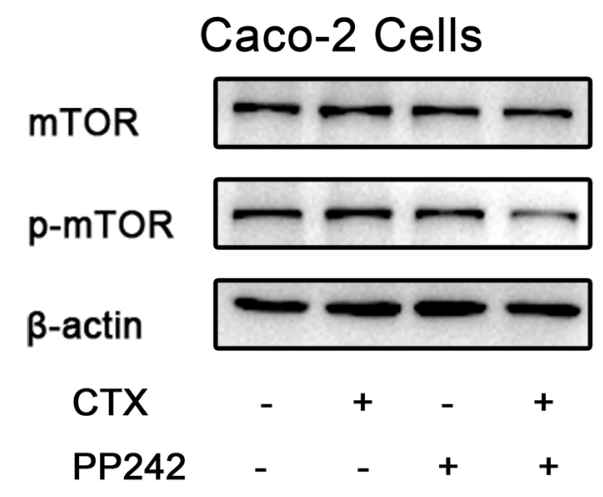

D

Caco-2 Cells

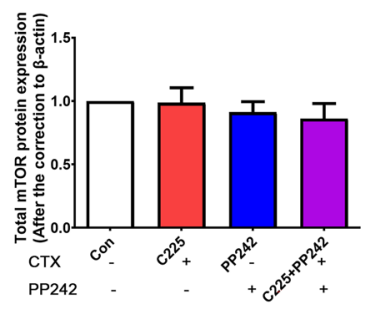

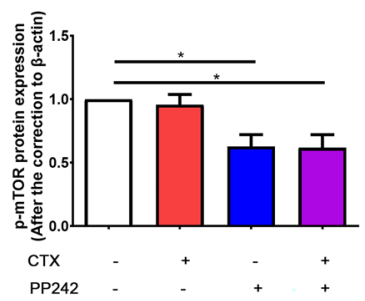

Figure S3 Application of PP242 inhibits the phosphorylation of mTOR in CRC cells. HT-29 cells and Caco-2 cells were untreated or treated with $20 \mu \mathrm{g} / \mathrm{mL}$ CTX and $1 \mu \mathrm{mol} / \mathrm{L}$ PP242 alone or in combination for $96 \mathrm{~h}$. (A,B) The protein content of cellular total and phosphorylated mTOR were determined by western blotting. (C,D) The semi-quantitative analysis of cellular total and phosphorylated mTOR contents was performed based on the relative density values of the bands of western blotting. The relative density value of the control group was set to one. Each bar represents the mean \pm SEM. All data were derived from at least three separate experiments. *, $\mathrm{P}<0.05$; **, $\mathrm{P}<0.01 ;{ }^{* * *}, \mathrm{P}<0.001$. mTOR, mammalian target of the rapamycin; CRC, colorectal cancer. SEM, standard error of mean. 